\title{
Using morphs of familiar objects to examine how shape discriminability influences view sensitivity
}

\author{
REBECCA LAWSON \\ University of Liverpool, Liverpool, England \\ AND \\ HEINRICH H. BÜLTHOFF \\ Max-Planck-Institut für biologische Kybernetik, Tübingen, Germany
}

\begin{abstract}
We investigated how the difficulty of detecting a shape change influenced the achievement of object constancy across depth rotations for object identification and categorization tasks. In three sequential matching experiments, people saw pictures of morphs between two everyday, nameable objects (e.g., bath-sink morphs, along a continuum between "bath" and "sink" end-point shapes). In each experiment, both view changes and shape changes influenced performance. Furthermore, the deleterious effects of view changes were strongest when shape discrimination was hardest. In our earlier research, using morphs of novel objects, we found a similar interaction between view sensitivity and shape sensitivity (Lawson, 2004b; Lawson \& Bülthoff, 2006; Lawson, Bülthoff, \& Dumbell, 2003). The present results extend these findings to familiar-object morphs. They suggest that recognition remains view-sensitive at the basic level of identification for everyday, nameable objects, and that the difficulty of shape discrimination plays a critical role in determining the degree of this view sensitivity.
\end{abstract}

One of the most impressive achievements of our visual system is its ability to abstract away from task-irrelevant variation in the input in order to identify and categorize objects. Our object recognition system is usually both fast and accurate at recognizing shapes across changes of retinal size, viewpoint, and illumination (see Lawson, 1999, for a review). This achievement of object constancy often, though, comes at a measurable cost in terms of the speed or the accuracy of processing. For example, if a familiar object is first seen at one view in depth and is subsequently shown at a different view, its identification in the second view is usually less efficient (so that priming is reduced), relative to when the second view of an object is identical or similar to the first (see, e.g., Hayward, 1998; Lawson \& Humphreys, 1996, 1998, 1999; Lawson, Humphreys, \& Watson, 1994; Srinivas, 1995; Thoma \& Davidoff, 2006; Vuilleumier, Henson, Driver, \& Dolan, 2002; see also Fang \& He, 2005, for similar results with an adaptation paradigm).

There is still no consensus as to the theoretical interpretation of these empirical findings of view-sensitive (and sometimes view-invariant) performance. However, the simplistic characterization of this debate as being between those arguing that object recognition is subserved only by 2-D representations finely tuned to viewpoint in depth and others proposing fully view-invariant 3 -D representations of objects has gradually evolved to cover a range of more complex and nuanced intermediate positions (see, e.g., Biederman, 1987; Biederman \& Gerhardstein, 1993, 1995; Bülthoff \& Edelman, 1992; Burgund \& Marsolek, 2000;
Demeyer, Zaenen, \& Wagemans, 2007; Foster \& Gilson, 2002; Hayward, 2003; Hayward \& Tarr, 1997; Hummel, 2001; Hummel \& Stankiewicz, 1998; Marr, 1982; Tarr, 1995; Tarr \& Bülthoff, 1995, 1998; Tarr \& Pinker, 1990; Thoma, Hummel, \& Davidoff, 2004; Tjan \& Legge, 1998; Vanrie, Willems, \& Wagemans, 2001; Vuilleumier et al., 2002; Wilson \& Farah, 2006).

One important obstacle to progress in understanding how the visual system achieves object constancy is the fact that the factors critical to determining the level of view sensitivity in a given situation still remain unclear. Many factors are likely to play a significant role. These include the class and structure of the stimuli to be identified (cf. the geometries of faces, animals, artifacts, and the twisted wire or block stimuli often used in studies of novel-object recognition); familiarity (both whether people have experience with the stimuli prior to testing and on the number of presentations of a given object within a study); task (performance may differ between initial recognition and short- or long-term priming, as well as across different tasks, such as picture-picture matching and naming); the difficulty of discriminating between objects (usually harder for face and subordinate-level object recognition and when many similarly shaped objects must be distinguished, relative to most instances of basiclevel object recognition); and stimulus presentation (e.g., whether real 3-D objects are seen-either monocularly or binocularly - or are presented as pictures on a computer monitor, and whether such depictions include color or shading, or are only line drawings).

R. Lawson, rlawson@liverpool.ac.uk 
All of these factors have been investigated in previous research into the achievement of object constancy. However, usually only one or two factors have been included within a single set of experiments. Furthermore, progress in this area has been hampered by difficulties in making cross-study comparisons, because of the diversity of both the tasks and the stimuli that have been used by different researchers. Few investigators have used either a fixed task with manipulations of different stimuli or multiple tasks with a standard set of stimuli. This means that the detection of systematic changes in view sensitivity due to interactions between factors has not been possible (Tarr \& Bülthoff, 1998).

We believe that it is essential to explore the pattern of view sensitivity across a wide set of conditions to gain a broad-based understanding of the effects of depth rotation on object recognition. A unique strength of the present study is that we use the same methodology as in earlier studies (Lawson, 2004b; Lawson \& Bülthoff, 2006; Lawson, Bülthoff, \& Dumbell, 2003), with a new set of stimuli. The results of the present study testing familiar objects, together with those of our previous studies testing novel objects, have allowed us to map out variations in view sensitivity across a wide set of conditions. Across these experiments, we manipulated the class and structure of the stimuli tested (using a large and geometrically diverse set of 3-D shapes from different superordinate categories), familiarity (across both novel and familiar, everyday objects), task (two recognition tasks and a categorization task), and, most importantly, the difficulty of the shape discrimination, which was varied within every study. Other differences between these studies were minimized: All used the same method of stimulus presentation (shaded grayscale pictures of objects presented briefly on a computer screen) and the same general methodology (speeded responses to a sequential picture-picture matching task). In ongoing research with 3-D versions of the familiar stimuli used in the present study, we are extending still further the set of conditions tested (Lawson, 2008).

\section{The Role of Shape Discriminability in Modulating the View Sensitivity of Object Recognition}

The main aim of the seven experiments here was to investigate, across a wide range of conditions, how the cost of achieving object constancy over rotation in depth is modulated by the difficulty of the shape discrimination required in a task. In particular, we have investigated whether view sensitivity is eliminated when shape discrimination is relatively easy. In a series of recent studies, we measured view sensitivity when people had to detect shape changes to novel objects (Lawson, 2004b; Lawson \& Bülthoff, 2006; Lawson et al., 2003). We found a striking interaction between view sensitivity and shape discrimination difficulty: When subtle shape changes had to be detected, performance was highly view-sensitive. In contrast, when only large shape changes had to be detected, performance was much less view-sensitive, and in some cases was even view-invariant. This pattern of performance generalized across three different experimental paradigms.

These findings support the claim that view sensitivity increases as the shape similarity of the objects that must be distinguished increases (Biederman \& Gerhardstein, 1993; Hamm \& McMullen, 1998; Tarr \& Cheng, 2003). This finding is relatively uncontroversial (but see Hayward \& Williams, 2000). In contrast, there is considerable disagreement about when object recognition becomes view-insensitive as shape discrimination becomes easier. For example, Biederman and Gerhardstein $(1993,1995)$ have argued that distinguishing between familiar objects at the basic level (e.g., identifying objects as exemplars of such categories as dogs, tables, and apples) is largely viewinvariant. In contrast, Tarr and colleagues (Tarr \& Bülthoff, 1995; Tarr \& Cheng, 2003) argued that such recognition is usually view-sensitive. The results of our earlier studies (Lawson, 2004b; Lawson \& Bülthoff, 2006; Lawson et al., 2003) have led us to support the latter claim, since we found view sensitivity even when shape discrimination seemed easier than that required for everyday object recognition. However, we wanted to test this claim more directly by using familiar rather than novel objects. This issuenamely, how changes in shape discriminability modulate view sensitivity for the identification of familiar objectsis the focus of the present article.

\section{The Role of Familiarity in Modulating the View Sensitivity of Object Recognition}

We present here the results from experiments in which we presented morphs of familiar objects (see Figures 1 and 2) and replicated the designs of three of our previous studies, which used morphed versions of novel objects. The familiar objects varied in shape in ways similar to how our novel object stimuli varied, allowing us to systematically manipulate shape discrimination difficulty in order to investigate its effect on view sensitivity. The use of familiar objects was critical, because it enabled us to deduce when object constancy over depth rotation can be achieved with minimal cost during our everyday experience with nameable objects, as well as to contrast performance for these stimuli with that for novel objects. Specifically, there are three reasons why the present study was necessary in order to extend our earlier findings.

First, our initial studies presented novel objects, so we did not know how the difficulty of shape discrimination for these stimuli compared with that required in everyday object recognition. If a finer level of identification were necessary to discriminate the novel objects than would be needed for the basic-level recognition of familiar objects, the discrimination of our novel objects might have been more like subordinate-level or face recognition. In this case, the view sensitivity that we observed for the novel objects would be consistent with most current theories of visual object recognition. Furthermore, if the recognition of the novel objects were like subordinate or face recognition, no conclusions could then be drawn from these results about view sensitivity in everyday, basic-level object recognition. We did not believe that this was the case, since our novel objects were carefully selected to be similar to basic-level objects (see Figure 1 of Lawson et al., 2003), but it was important to test this possibility.

Second, although the novel objects presented in our earlier studies were chosen to be physically similar to familiar 


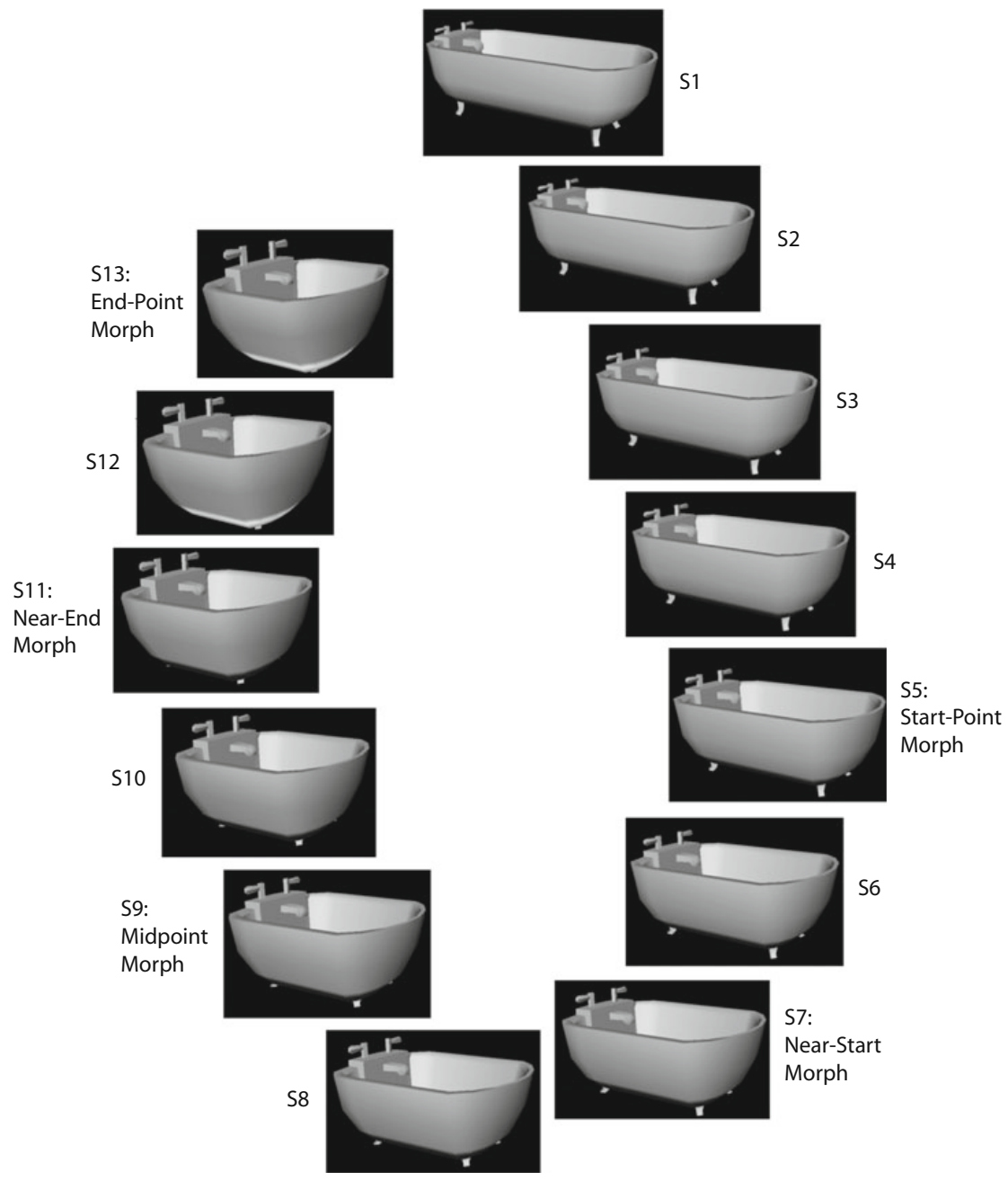

Figure 1. The $60^{\circ}$ view of all 13 morphs of 1 of the 20 experimental morph sets, bath-sink. The S1, S7, and S13 morphs were used in Experiment 1. In all subsequent experiments, only morphs between the start-point (here S5, a bath) and end-point (here S13, a sink) morphs were presented. The start- and end-point morphs were selected to equate the ease and consistency of identification of these two morphs and so that the midpoint morph (here, S9) would be identified about equally often with the start-point name ("bath") and the end-point name ("sink"). The start-point, near-start, midpoint, near-end, and end-point morphs were the S1, S4, S7, S10, and S13 morphs, respectively, for 5 of the morph sets; the S1, S3, S5, S7, and S9 morphs, respectively, for 1 morph set; and the S5, S7, S9, S11, and S13 morphs, respectively, for 14 morph sets, including the bath-sink set illustrated here. See Experiments 1 and 5 for further details.

objects, they might have differed on important shape dimensions. For example, the structure, symmetry, and complexity of the novel objects might have differed from that of most familiar objects. Any such confounding factors could, in turn, have influenced the degree of view sensitivity observed for these novel stimuli. For instance, view sensitivity could have been greater for the novel objects because they were more complex or less symmetrical than most familiar objects.

Third, familiarity per se may influence view sensitivity (for work on this topic with faces, see Eger, Schweinberger, Dolan, \& Henson, 2005; F. Jiang, Blanz, \& O'Toole, 2007; Pourtois, Schwartz, Seghier, Lazeyras, \& Vuilleumier,
2005; Ryu \& Chaudhuri, 2006; and the work summarized below). For example, view sensitivity may be greater for novel than for familiar objects because of differences in their stored visual representations. As people gain visual experience with a category of objects by seeing different views of multiple exemplars, they may gradually acquire stored, view-invariant representations of objects in that category. Alternatively, with experience they may acquire multiple view-specific representations of objects from a given category, and all of those representations may be activated when an object from that category is encountered. A third possibility is that, relative to novel objects, view 


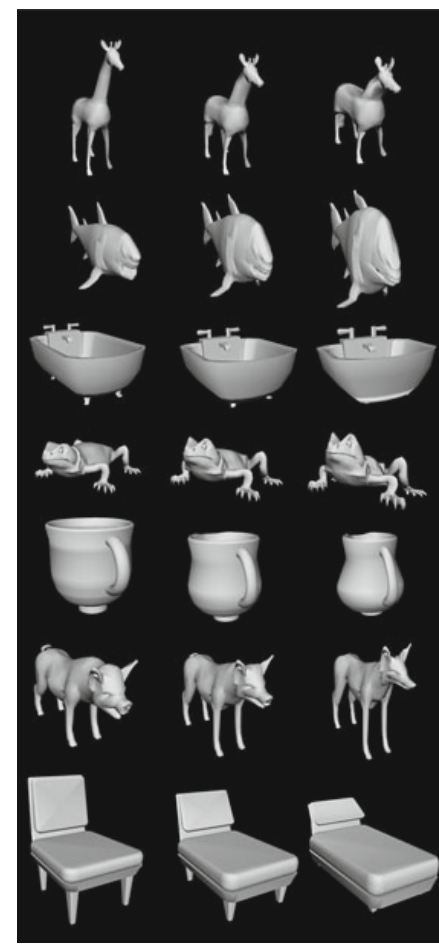

Giraffe-Horse

Shark-Fish

Bath-Sink

Lizard-Frog

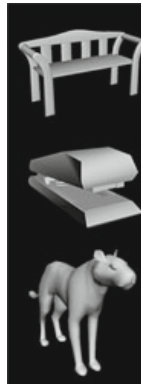

$\mid \frac{1111}{1}$

iㅔ

Bench-Chair

Cup-Jug

Pig-Dog
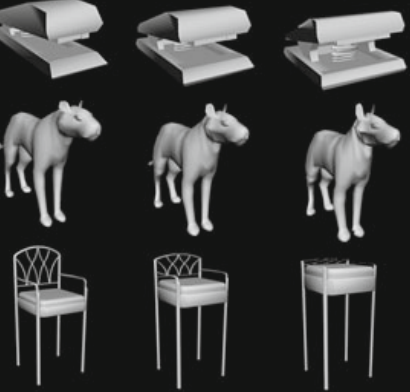

Stapler-Holepunch

Lion-Dog
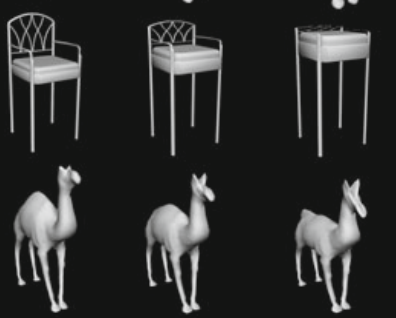

Chair-Stool

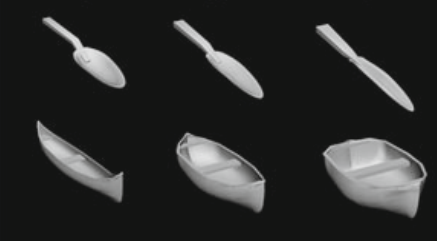

Camel-Llama

Chair-Bed

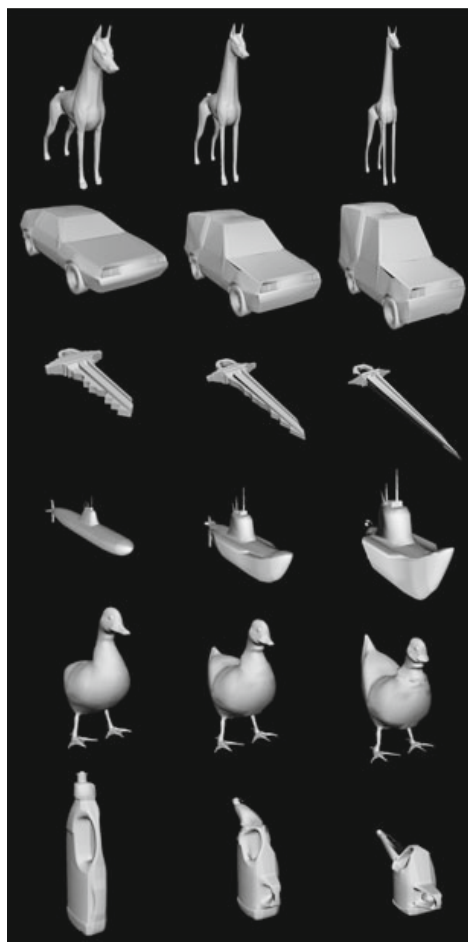

Dog-Giraffe

Car-Van

Spoon-Knife

Canoe-Rowing boat

\section{Car-Van \\ Key-Sword}

Submarine-Boat

Duck-Hen

Bottle-Wateringcan

Figure 2. The $30^{\circ}$ view of the start-point (left), midpoint, and end-point (right) morphs from each of the 20 experimental morph sets used in Experiments 4, 5, 6, and 7. The modal names of the start- and end-point morphs are given to the right of each trio of pictures for each morph set. 
sensitivity may be reduced for familiar objects because they can be named and have stored semantic knowledge associated with them. The recognition of familiar objects may then be partly mediated by view-invariant verbal or semantic processing. In the present study, we tested discrimination between differently shaped morphs that would usually be given the same name (Experiment 5) and categorization of pairs of morphs that would usually be given different names (e.g., baths and sinks; Experiment 7).

Indirect evidence that familiarity may play an important role in the view sensitivity of object recognition comes from a plethora of recent research with faces, using both adaptation and imaging methodologies. These studies have reported a number of differences between performance with familiar versus unfamiliar faces. Their results are not fully consistent with each other and are open to different interpretations. Nonetheless, they do point to familiarity playing an important role in modulating view sensitivity for faces.

Adaptation studies have demonstrated that face identification is highly sensitive to view in depth. Behavioral studies have reported decreased adaptation as the angle between the adapting and the test stimulus increases (e.g., Benton, Jennings, \& Chatting, 2006), and view-specific face adaptation has been reported in fMRI imaging studies (e.g., Andrews \& Ewbank, 2004; Grill-Spector et al., 1999). Using an identity adaptation paradigm, F. Jiang et al. (2007) found that adaptation was greater when the adapting and test faces were shown from the same view rather than different views for both familiar and unfamiliar faces. The researchers also concluded that face familiarity enhanced the transferability of adaptation effects across views in depth (so that familiar face recognition was less view-sensitive). However, although adaptation effects were greater for familiar faces in their study, there was not a significant interaction between familiarity and the sensitivity of adaptation to view changes. Ryu and Chaudhuri (2006) investigated orientation adaptation effects for faces. In their first experiment, they found greater visual adaptation to the orientation of familiar than of unfamiliar faces. This suggests that adaptation to the orientation of familiar faces is more view-sensitive. In their second experiment, they found very poor orientation discrimination for a familiar face following adaptation with a different familiar face, whereas for unfamiliar faces, between-face adaptation was similar to within-face adaptation. Here, showing one face for 5 sec disrupted people's ability to detect the orientation of a different face if both faces were familiar, but not if both faces were unfamiliar. This surprising result is difficult to interpret, and further research on this topic seems necessary before clear conclusions can be drawn.

Imaging studies have also provided evidence that face identification is influenced by both the view in depth at which a face is presented and the familiarity of that face. Eger et al. (2005) investigated the effect of familiarity using a sex decision task. They reported similar levels of image-specific priming for familiar and unfamiliar faces in their behavioral data. In contrast, they found greater image specificity for familiar (but not for unfamiliar) faces in activity in the anterior relative to the middle fusiform region. However, Eger et al.'s visual change condition had an uncontrolled mix of changes, including some depth ro- tations, but also alterations to lighting, facial expression, hairstyles, and so on. This means that it is not possible to specify which changes produced the differences that they observed. A similar study was reported by Pourtois et al. (2005). As in Eger et al.'s study, the differences here between the two test images of a person's face were relatively unconstrained and included alterations to age and appearance as well as to the view in depth. Unlike Eger et al., Pourtois et al. found no behavioral effects in their sex decision task, perhaps because of ceiling performance. In their imaging results, they found evidence for repetition priming across visual changes for both familiar and unfamiliar faces, but the locations of these effects differed. Note, too, that unlike Eger et al., they did not run a control, same-view condition, so the differences that they observed between familiar and unfamiliar faces may not have been specific to the achievement of object constancy.

Differences in the designs and results of these studies make it difficult to draw strong conclusions about the view sensitivity of familiar as compared with unfamiliar face identification. However, the overall pattern of findings demonstrates that familiarity may play a major role in modulating view specificity for face recognition. For this reason, it is clearly important to examine whether the view sensitivity of object recognition is also influenced by familiarity. In particular, we chose to investigate whether, when tested in comparable conditions, the recognition of familiar objects was much less view-sensitive than that of novel objects.

\section{Stimulus Selection}

In order to draw valid conclusions about the recognition and categorization of familiar objects, it was critical to select appropriate stimuli. We therefore conducted an initial series of four experiments to allow us to choose suitable pairs of familiar objects (Experiments 1 and 2), select a range of morphs between those objects (Experiment 1), and verify that the extremes of this range (the start-point and end-point morphs) would be identified using the intended names (Experiment 4). We also collected typicality ratings for the names of the start- and end-point morphs (Experiment 3), as well as asking people whether they believed that objects in between these categories could exist and what common label could be applied to each pair of start- and end-point names (Experiment 3).

Note that morphs between the start and end points were not intended to be familiar to people. These morphs would rarely be similar to objects encountered in everyday life, and people were not expected to be able to confidently or consistently name them. Instead, the participants would often be torn between giving these in-between morphs the labels of the start-point and end-point categories, with the object being a poor exemplar of both. These in-between morphs were created because it is difficult to find sequences of familiar objects that vary systematically in shape and have different start- and end-point labels: One example would be a tadpole growing to an adult frog.

The initial experiments ensured that the stimuli presented in the three final experiments comprised sets of morphs that spanned the shape space between fairly typical exemplars of two familiar, nameable categories of objects (e.g., 
bath-sink; see Figure 1). Within each of these morph sets, the initial, start-point morph was usually given one name (e.g., $100 \%$ of naming responses were "bath"); the final, end-point morph was normally given a different name (e.g., $84 \%$ "sink" responses); and the midpoint morph was identified using an approximately equal mix of the start- and endpoint names (e.g., 66\% "bath" and 32\% "sink" responses). These were exacting conditions for stimulus selection, so we began with a large set of 53 pairs of objects. From the 53 sets of morphs generated between each of these pairs, the 20 best sets were selected, and the most suitable start-point, midpoint, and end-point morphs within each sequence were chosen (see Figure 2). These stimuli were then presented in three sequential picture-picture matching experiments in which we tested object recognition (Experiments 5 and 6 ) and categorization (Experiment 7) while varying the difficulty of shape discrimination.

\section{EXPERIMENT 1}

Participants named depth-rotated views of morphs spanning the range of shapes between each of the 53 pairs of familiar objects. These results were used to select morph sets for which the start-point and end-point morphs were each named consistently and with different names.

\section{Method}

Participants. Thirty-six undergraduate students from the University of Liverpool took part in exchange for course credit.

Materials for all experiments. Fifty-three 3-D models of familiar objects were selected: These were the $\mathrm{S} 1$ morphs. The shape of each $\mathrm{S} 1$ morph was then changed incrementally to produce a series of 12 more morphs, labeled S2 to S13. The S13 morph had the shape least similar to S1 and was intended to resemble a different familiar object. The $60^{\circ}$ views for the bath-sink morph set are shown in Figure 1. Pictures of 12 depth-rotated views were produced for each of these 13 morphs. The $0^{\circ}$ view was assigned to be a foreshortened view if the object had an elongated shape, or was otherwise arbitrarily assigned. Foreshortened views can be particularly difficult to identify (Lawson, 1999; Lawson \& Humphreys, 1999), so we avoided presenting them. From the $0^{\circ}$ view, each successive view was rotated by $30^{\circ}$ about the vertical axis running through the midpoint of the morph. These views were labeled as $30^{\circ}, 60^{\circ}, 90^{\circ}$, and so on, up to $330^{\circ}$. Altogether, there were 156 pictures ( 13 morphs, each shown from 12 views) of each of the 53 morph sets. Grayscale images of the views of the morphs were generated using the SoftImage rendering software. The images were presented against a black background inside a window measuring $135 \times 110 \mathrm{~mm}$ on a computer monitor, at a viewing distance of approximately $50 \mathrm{~cm}$.

Design and Procedure. The S1, S7, and S13 morphs from each set were used. Each morph was depicted from four views in depth: $30^{\circ}, 60^{\circ}, 210^{\circ}$, and $240^{\circ}$. Thus, in total 12 pictures from each morph set were presented. All participants completed one block of 159 trials in which the S1, S7, and S13 morphs from the 53 morph sets were each seen once. Four groups of 9 participants were shown different views of the morphs: Two saw a mixture of $30^{\circ}$ and $240^{\circ}$ views, and the other two saw a mixture of $60^{\circ}$ and $210^{\circ}$ views. Across the four groups, all views of each of the morphs were shown an equal number of times.

The experiment was run on a Macintosh PowerPC G4 computer using PsyScope (version 1.2.5) experimental presentation software. On each trial, a picture was presented centrally, and participants were cued to type in the name of the object. They were not encouraged to make speeded responses. The trials were presented in a different random order to each participant, and the experiment lasted around $30 \mathrm{~min}$.

\section{Results and Discussion}

Spelling mistakes were corrected, shortened forms were replaced with standardized responses (e.g., "bike" was changed to "bicycle"), and multiword responses were reduced to a single word (e.g., "wide chair" was replaced by "chair"). If two alternative names were provided (e.g., "horse/ giraffe"), the second was deleted. The percentage choice of the modal name for each S1, S7, and S13 morph (averaged over the four views tested) was calculated. The overall percentages of modal name choice were similar for each of the four separate views of the S1, S7, and S13 morphs.

Five of the 53 morph sets were eliminated at this stage because they were not named consistently. The remaining 48 morph sets were tested in a word-picture verification task in Experiment 2. In Experiment 2, people selected one of three alternative names for each picture of a morph from a given set. These names were chosen on the basis of the results of Experiment 1. They comprised the modal names given to the $\mathrm{S} 1$ and $\mathrm{S} 13$ morphs from that set (e.g., "key" and "sword" for the key-sword morph set), plus another common name given to pictures from that morph set ("saw" for the key-sword morph set). For the 20 morph sets selected as the stimuli in Experiments 5, 6, and 7, the overall accuracy of naming was $86 \%$ for the S1 morphs (5\% of participants gave the S13 name and $10 \%$ another name) and $70 \%$ for the $\mathrm{S} 13$ morphs (5\% gave the $\mathrm{S} 1$ name and $23 \%$ another name).

The results from Experiment 1 were also used to select which morphs were assigned as the start-point, midpoint, and end-point morphs for a given morph set in all of the subsequent experiments. The default assignment was S1 as the start-point, S7 as the midpoint, and S13 as the endpoint morph. This assignment was used for 5 of the 20 morph sets that were chosen as the experimental stimuli. However, if the S1 (or S13) morph dominated naming in Experiment 1, different morphs were selected, in an attempt to equate the proportions of start-point and endpoint names assigned to the midpoint morph and equate the accuracies of naming for the start-point and end-point morphs. For 14 of the morph sets (including the bath-sink set illustrated in Figure 1), the S7 morphs were mostly given the start-point name. For these morph sets, the revised start-point morph was $\mathrm{S} 5$, and the revised midpoint morph was S9. For 1 morph set (cup-jug), S7 morphs were mostly given the end-point name, so the revised end-point morph was S9 and the revised midpoint morph was S5.

\section{EXPERIMENT 2}

In Experiment 2, a word-picture verification task was used to check the preferred names for the start-point morphs (S1 or S5), midpoint morphs (S5, S7, or S9), and end-point morphs (S13 or S9) for the 48 sets selected in Experiment 1. Experiment 1 placed a lower bound on the consistency of naming the morphs. However, in Experiment 1 people may have recognized a given object but been unable to recall the most appropriate name, or they may have used different names to refer to identical or similar shapes (such as a deer, stag, antelope, or moose). In Experiment 2, the upper bound of naming consistency was tested by asking people 
to choose one label from just three alternative names with which to identify each morph. Experiment 2 also tested whether the midpoint morph was approximately at the category boundary (i.e., whether it was labeled with similar proportions of start-point and end-point names).

\section{Method}

Participants. Thirty-two undergraduate students from the University of Liverpool took part in exchange for course credit.

Materials, Design, and Procedure. For each of the 48 morph sets selected from Experiment 1, the same three written names were shown with all of the morphs from a given set. These names were the modal responses to the S1 and S13 morphs in Experiment 1, plus a common alternative response given to morphs from that morph set; see Table 1. In addition, participants could select "other" to indicate that they would label an object with a name that did not appear as one of the options provided. If they chose this response, they were asked to type in their preferred name. The start-point, midpoint, and end-point morphs used were those selected for each morph set in Experiment 1. Each of these morphs was, in turn, depicted in $30^{\circ}$, $60^{\circ}, 90^{\circ}$, and $120^{\circ}$ views.

Each participant completed two blocks of 144 trials. Across these trials, morphs from each of the 48 sets were presented six times: once from each of two views for each of the three morphs. For half of the participants, the two views presented were $30^{\circ}$ and $120^{\circ}$, and for the remaining participants, they were $60^{\circ}$ and $90^{\circ}$. The six possible arrangements of positions (left, middle, and right) of the startpoint, end-point, and alternative names were each used once for the six presentations of morphs from a given set for every participant. Across all participants, each view of all of the morphs was shown an equal number of times.

The experiment was run on a Macintosh PowerPC G4 computer using PsyScope (version 1.2.5) experimental presentation software. On each trial, a picture of a morph was presented centrally and three numbered names were presented below it, plus a fourth, "other name" option. Participants made an unspeeded keypress response to select one of the names, or they pressed "4" to select "other name" and then typed in their preferred name. The trials were presented in a different random order to each participant, and the experiment lasted around $20 \mathrm{~min}$.

\section{Results and Discussion}

Reassuringly, the start-point name dominated responses to the start-point morph (92\%) and the end-point name dominated responses to the end-point morph (68\%). People chose the start-point name $(92 \%, 60 \%$, and $22 \%$ for the start-point, midpoint, and end-point morphs, respectively) more often than the end-point name $(5 \%, 32 \%$, and $68 \%)$. They rarely chose the alternative name $(2 \%, 8 \%$, and $9 \%)$, and very few provided their own name for a stimulus (less than $1 \%$ for each morph).

Responses for each of the 48 morph sets were examined separately in order to select sets for which the startpoint morph was usually given the start-point name, the end-point morph was usually given the end-point name, and the midpoint morph was usually given a mixture of the start-point and end-point names. For 25 morph sets, both the start-point and the end-point morphs were assigned their modal name on at least $50 \%$ of the trials. Twenty of these sets were chosen to be experimental morph sets by selecting to maximize object diversity (see Table 1). For these sets, $89 \%$ of people chose the start-point name for the start-point morph (minimum $69 \% ; 7 \%$ chose the end-point name, and $3 \%$ chose the alternative name). For the end-point morph, $83 \%$ chose the end-point name (minimum 59\%; 11\% chose the start-point name, and 5\% chose the alternative name). For the midpoint morph, $47 \%$ chose the start-point name and $45 \%$ the end-point name (minimum of $16 \%$ in both cases; $7 \%$ chose the alternative name).

Table 1

Percentage of Selection of the Start-Point, End-Point, and Third Alternative Names for the Start-Point, Midpoint, and End-Point Morphs in the Word-Picture Verification Task in Experiment 2, Plus the Percentages of Self-Generated Names Averaged Across All Three Morphs, for the 20 Experimental Morph Sets Selected for Use in Subsequent Experiments

\begin{tabular}{|c|c|c|c|c|c|c|c|c|c|c|c|c|c|}
\hline \multirow[b]{2}{*}{$\begin{array}{l}\text { Morph } \\
\text { Group }\end{array}$} & \multirow[b]{2}{*}{$\begin{array}{l}\text { Start- } \\
\text { Point } \\
\text { Name }\end{array}$} & \multicolumn{3}{|c|}{ Start-Point Morph } & \multicolumn{3}{|c|}{ Midpoint Morph } & \multicolumn{3}{|c|}{ End-Point Morph } & \multirow[b]{2}{*}{$\begin{array}{c}\text { End-Point } \\
\text { Name }\end{array}$} & \multirow[b]{2}{*}{$\begin{array}{l}\text { Other } \\
\text { Name }\end{array}$} & \multirow[b]{2}{*}{$\begin{array}{c}\text { Self- } \\
\text { Generated } \\
\text { Names } \\
\end{array}$} \\
\hline & & $\begin{array}{l}\text { Start- } \\
\text { Point } \\
\text { Name }\end{array}$ & $\begin{array}{l}\text { Other } \\
\text { Name }\end{array}$ & $\begin{array}{l}\text { End- } \\
\text { Point } \\
\text { Name }\end{array}$ & $\begin{array}{l}\text { Start- } \\
\text { Point } \\
\text { Name }\end{array}$ & $\begin{array}{l}\text { Other } \\
\text { Name }\end{array}$ & $\begin{array}{l}\text { End- } \\
\text { Point } \\
\text { Name }\end{array}$ & $\begin{array}{l}\text { Start- } \\
\text { Point } \\
\text { Name }\end{array}$ & $\begin{array}{l}\text { Other } \\
\text { Name }\end{array}$ & $\begin{array}{l}\text { End- } \\
\text { Point } \\
\text { Name }\end{array}$ & & & \\
\hline 1 & GIRAFFE & 95 & 2 & 3 & 52 & 2 & 45 & 6 & 6 & 84 & HORSE & DOG & 2 \\
\hline 1 & SHARK & 97 & 2 & 2 & 72 & 0 & 28 & 31 & 3 & 66 & FISH & DOLPHIN & 0 \\
\hline 1 & BATH & 97 & 0 & 3 & 75 & 2 & 23 & 5 & 0 & 95 & SINK & SHOWER & 0 \\
\hline 1 & LIZARD & 89 & 3 & 5 & 59 & 2 & 38 & 39 & 2 & 59 & FROG & CROCODILE & 1 \\
\hline 1 & CUP & 80 & 0 & 20 & 17 & 8 & 75 & 2 & 8 & 91 & JUG & VASE & 0 \\
\hline 1 & PIG & 98 & 0 & 2 & 56 & 2 & 41 & 6 & 2 & 80 & DOG & COW & 3 \\
\hline 1 & CHAIR & 86 & 6 & 6 & 47 & 9 & 41 & 2 & 2 & 95 & BED & SOFA & 2 \\
\hline 1 & BENCH & 98 & 2 & 0 & 38 & 9 & 53 & 5 & 0 & 95 & CHAIR & STOOL & 0 \\
\hline 1 & STAPLER & 81 & 0 & 17 & 47 & 0 & 53 & 19 & 2 & 80 & HOLEPUNCH & LAMP & 0 \\
\hline 1 & LION & 83 & 3 & 14 & 56 & 6 & 36 & 17 & 5 & 78 & DOG & CAT & 0 \\
\hline 2 & CHAIR & 69 & 2 & 30 & 38 & 2 & 61 & 14 & 6 & 78 & STOOL & BENCH & 0 \\
\hline 2 & CAMEL & 83 & 0 & 17 & 33 & 0 & 67 & 5 & 3 & 92 & LLAMA & HORSE & 0 \\
\hline 2 & SPOON & 75 & 25 & 0 & 19 & 38 & 42 & 0 & 13 & 88 & KNIFE & TROWEL & 0 \\
\hline 2 & CANOE & 95 & 0 & 5 & 39 & 0 & 61 & 8 & 8 & 84 & ROWING BOAT & RAFT & 0 \\
\hline 2 & DOG & 95 & 2 & 2 & 64 & 0 & 36 & 2 & 2 & 97 & GIRAFFE & HORSE & 0 \\
\hline 2 & CAR & 98 & 0 & 0 & 16 & 3 & 80 & 3 & 5 & 92 & VAN & LORRY & 1 \\
\hline 2 & KEY & 84 & 16 & 0 & 47 & 36 & 16 & 3 & 2 & 94 & SWORD & SAW & 1 \\
\hline 2 & SUBMARINE & 98 & 0 & 2 & 72 & 0 & 28 & 31 & 3 & 63 & BOAT & YACHT & 0 \\
\hline 2 & DUCK & 95 & 0 & 3 & 50 & 13 & 36 & 14 & 23 & 63 & CHICKEN & PIGEON & 0 \\
\hline \multirow[t]{2}{*}{2} & BOTTLE & 95 & 0 & 2 & 28 & 3 & 66 & 2 & 0 & 97 & WATERINGCAN & JUG & 2 \\
\hline & Mean & 89 & 3 & 7 & 47 & 7 & 45 & 11 & 5 & 83 & & & 1 \\
\hline
\end{tabular}




\section{EXPERIMENT 3}

Rating data were collected from three groups of participants for the 20 experimental morph sets selected in Experiment 2. The first group rated the typicality of the start-point and end-point modal names for the start-point and end-point morphs. This was used to ensure that, for the start-point morphs, the start-point name was considered to be an appropriate label but the end-point name was not, and vice versa for the end-point morphs. This provided a final check of the appropriateness of the labels selected for these stimuli in Experiments 1 and 2.

The second rating group decided whether an object could exist that was midway between the start-point and end-point categories for a given morph set. These data were used to check whether people thought that objects shaped like the midpoint morphs could (or already) exist in the world. If so, the midpoint morph could be considered either to be a typical exemplar of the start-point or the end-point category or to have a different but appropriate label. In either case, the midpoint morph itself would be a familiar object. For example, people's stored visual representations of plates and bowls are likely to overlap with each other in shape space. If so, people might consider either "plate" or "bowl" to be an acceptable label for deep plates and shallow bowls. We instead intended a midpoint morph to be sufficiently distinct from either its start-point or end-point morph that it would be a poor exemplar of either category.

The third rating group provided a label that could apply to both the start-point and end-point categories for a given morph set (e.g., "animal" for the pig-dog set). Together with the results from Experiments 1 and 2, which investigated the preferred names given to the morphs, this provided evidence about the level of identification (basic or subordinate) of objects in a given morph set. The assignment of a given label for a category of objects to the superordinate, basic, or subordinate level cannot be determined a priori and appears to be knowledge-dependent (Medin \& Atran, 2004). Few researchers have collected converging measures across different tests to establish the category level of a given name (see Lawson \& Jolicœur, 2003; see also Joliceur, Gluck, \& Kosslyn, 1984), so this information was not available for many of our start-point and endpoint names. However, our population was similar to that tested by Rosch, Mervis, Gray, Johnson, and Boyes-Braem (1976), so we could use the levels established by them for some items. We also reasoned that, if the common label provided by raters was one generally agreed to be at the superordinate level (e.g., "animal," "furniture," "vehicle"), this would indicate that the separate start- and end-point labels subsumed under it were basic-level labels. However, if the common label was usually considered to be a basic-level label by our undergraduate population (e.g., "bird," "boat," "fish"), this would suggest that the startand end-point labels were at the subordinate level.

\section{Method}

Participants. The first (typicality) and third (common-label) rating groups consisted of 24 and 16 undergraduate students, respectively, from the University of Liverpool, who took part for course credit. The second (existence) rating group comprised 61 prospective undergraduate students and their parents.

Materials, Design, and Procedure. Participants in the typicality rating group completed 80 trials in which they rated $30^{\circ}$ views of morphs in terms of how good an example they were of a named category. The start- and end-point morphs from the 20 experimental morph sets were each shown twice to a participant, once paired with the start-point name and once with the end-point name. The start-point morph was shown before the end-point morph for 10 of the morph sets, and vice versa for the other 10 sets. For 5 in each subset of 10 morph sets, a morph was paired with the start-point name before being paired with the end-point name, and vice versa for the other 5. The order of assignment of morph sets to these four presentation conditions was counterbalanced across four groups of 6 participants. Participants rated each morph for how good an example of the named category it was, using a scale from 1 (a very good example of their idea or image of the labeled category of objects) to 7 (a very poor example)

Participants in the existence rating group and the common-label rating group each completed 20 trials. On each trial, pairs of words were shown, comprising the start- and end-point names for each of the 20 experimental morph sets. Note that no pictures of the morphs were presented. For half of the word pairs, the start-point name was presented to the left of the end-point name, and vice versa for the other half.

The existence rating group decided whether objects halfway between the two named categories could exist. They were told that, for example, if the two words were "trousers" and "shorts," objects halfway between would almost certainly exist in the real world. However, if the two words were "bottle" and "sieve," probably nothing that is half-bottle, half-sieve would exist in the world. For each pair of labels, participants circled a number between 1 and 5 to indicate whether the halfway objects (1) probably exist in the world right now, (2) possibly might exist in the world right now, (3) could never exist but I can imagine what it might look like, (4) could never exist and I cannot even imagine what it might look like, or (5) other. If they selected 5, they were asked to write their own response.

The common-label task was run after participants had completed Experiment 2, so these participants had seen pictures of the objects, although they were not told that the two experiments were related. The participants were asked to provide a category label that included both the start-point and end-point object categories for a given morph set, such that the object categories would both be "kinds of" the label that they chose. As examples, participants were told that Stilton and Brie were both kinds of cheese, cake and onion were both kinds of food, and oak and apple were both kinds of tree.

\section{Results and Discussion}

Typicality rating group. Ratings ranged from 1 (for a prototypical exemplar of a category) to 7 (for a poor exemplar or nonmember of a category). For start-point morphs, the start-point name was rated as more typical (2.4; range 1.5-3.9) than the end-point name (5.1; range 3.3-6.4); see Table 2. For the end-point morphs, the end-point name was rated as more typical (2.9; range 1.4-4.4) than the start-point name (5.1; range 3.3-6.5). This confirmed the results of Experiment 2: The start-point morph was rated as a much better exemplar of the category labeled by the start-point than by the end-point name, and vice versa for the end-point morph.

Existence rating group. In most cases, people did not believe that an item midway between the start- and end-point labels could ever exist $(60 \%$ of responses were 3 or 4), though some midway items were thought to possibly exist (25\%) or to already exist (14\%). Participants were less likely to state that a midway item could exist for the eight animals (mean rating $=3.0$ ) than for the 10 artifacts (2.2). This difference was significant in a by-items ANOVA 


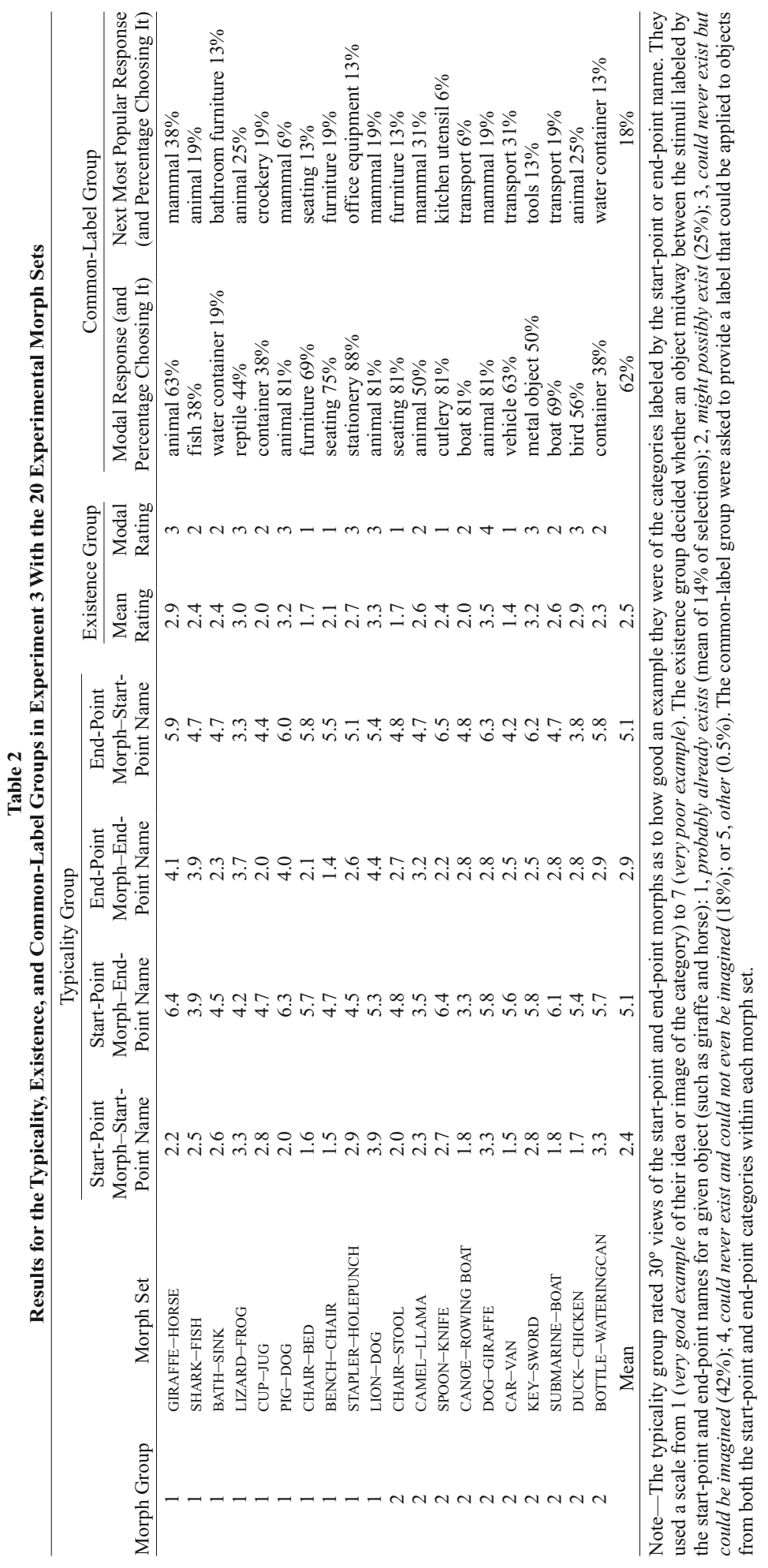


$[F(1,18)=13.936, p<.002]$. This result is consistent with the claim that people believe that some true, defining features are necessary and sufficient for all members of a biological category to possess, but that such psychological essentialism does not extend to artifacts (Gelman, 2003; Keil, 1989, 2003; Murphy, 2002). Note, though, that even for the artifacts, in most cases people did not believe that objects labeled by the start-point name were on a shape continuum with objects labeled by the end-point name. Instead, these objects were generally believed to belong to distinct categories, with no intermediate objects existing between them. This implies that the midmorphs for these morph sets would be viewed as unfamiliar and highly atypical exemplars of both the start- and end-point categories. The clearest exceptions were for the furniture (chair-bed, bench-chair, and chair-stool), spoon-knife, and car-van morph sets.

Common-label rating group. There were five morph sets for which the superordinate label "animal" (or the less common alternative "mammal") was provided consistently as the common label: giraffe-horse, pig-dog, lion-dog, camel-llama, and dog-giraffe. The start- and end-point names for these morph sets would therefore generally be agreed to be basic-level labels. Other examples for which the modal common label seemed to be at the superordinate level were cup-jug ("container" or "crockery"), chair-bed ("furniture"), stapler-holepunch ("stationery"), spoon-knife ("cutlery"), car-van ("vehicle"), and bottle-wateringcan ("container"). For two further morph sets (bath-sink and key-sword), little agreement emerged as to a suitable common label, suggesting that these concepts are so distinct that they are not easily grouped into the same superordinate category. We therefore believe that these start- and end-point names should also be considered to be at the basic level. Altogether, 13 morph sets therefore appeared to have basic-level start- and end-point names.

The remaining seven morph sets were mostly given basic-level common labels, so the start- and end-point names for these sets would probably be considered to be subordinate-level labels. However, in all cases, the second most frequently provided label for these morph sets was at the superordinate level. These sets were shark-fish (the basic-level label "fish" was the modal common label, but the superordinate-level label "animal" was the next most frequent response), lizard-frog ("reptile," then "animal"), duck-chicken ("bird," then "animal"), bench-chair ("seating," then "furniture"), chair-stool ("seating," then "furniture"), canoe-rowing boat ("boat," then "transport"), and submarine-boat ("boat," then "transport").

Overall, these results suggest that most of the preferred start- and end-point names for the 20 experimental morph sets were at the basic level, although around a third were at the subordinate level. Most importantly, as we will show later, there was no evidence that view effects in Experiments 5 and 6 were greater for the 7 morph sets with subordinate labels (with a mean advantage for same-view over view-change trials of $145 \mathrm{msec}$ and $19 \%$ for errors), as compared with the 13 morph sets with basic-level labels (mean advantage for same-view trials $128 \mathrm{msec}$ and $18 \%$ errors; see Table 5). Indeed, the only morph set that produced no overall view effects (chair-stool) had start- and end-point names that were subordinate-level labels. There was, therefore, no evidence that the view effects that we observed in the subsequent experiments were driven by the minority of items that were identified at the subordinate level (as opposed to by the difficulty of shape discrimination).

\section{EXPERIMENT 4}

Experiment 4 provided a final confirmation of the choice of experimental stimuli. Three large groups of participants were tested with a free naming task. As in Experiment 1, we tested which labels were assigned to morphs. However, in Experiment 1 the S1, S7, and S13 morphs were shown, whereas different morphs (i.e., the ones selected in Experiment 2) were presented in Experiment 4 for most morph sets. It was important to check the consistency and appropriateness of the labels given to the start-point, midpoint, and end-point morphs for the experimental sets, since they were the stimuli that were presented in the subsequent experiments. In addition, the large number of participants tested in Experiment 4 provided good estimates of the proportions of start- and endpoint names used to label the midpoint morphs.

\section{Method}

Participants. Three groups of 142, 133, and 175 prospective undergraduate students and their parents, who were visiting the University of Liverpool for an open day, volunteered to take part in the experiment.

Materials, Design, and Procedure. Two of the groups each completed two blocks of 20 trials. In each block, people saw one morph from each of the 20 morph sets. In the first block, they saw the start-point morphs for 10 morph sets and the end-point morphs for the other 10, and the assignment of the start- or end-point morph for a given set was counterbalanced across the two groups. In the second block, both groups saw the midpoint morphs of all 20 morph sets. The third group only completed this second block of 20 trials; their results were used to check the names given to the midpoint morphs when recognition had not been primed by seeing either a start- or end-point morph, as was the case for the other two groups. All morphs were depicted from the $30^{\circ}$ view and were projected onto a large screen in a lecture theater. On each trial, a picture was presented for approximately $5 \mathrm{sec}$, and participants were asked to write down in a booklet the name of the pictured object.

\section{Results and Discussion}

Spelling mistakes were corrected, abbreviations were replaced with standardized responses, and multiword responses were reduced to a single word. If two alternative names were provided, the second was deleted. No response was made on $1.5 \%$ of trials, and responses were indecipherable on $0.2 \%$ of the trials.

The percentage choices of the modal names provided for the start-point, midpoint, and end-point morphs were calculated separately for each morph set; see Table 3. Any alternative names given by more than $5 \%$ of the participants are listed in Table 4, together with the names that were considered equivalent to other names, such as "pony" for "horse." Averaged across all 20 sets, $88 \%$ gave the startpoint name for the start-point morph (minimum $45 \%$; $5 \%$ gave the end-point name, and $6 \%$ gave other names). For the end-point morph, $75 \%$ gave the end-point name (minimum 17\%; 7\% gave the start-point name, and 19\% 
Table 3

Percentages of Start-Point and End-Point Names Provided for the Start-Point, Midpoint, and End-Point Morphs in the Naming Task in Experiment 4, Plus the Percentages of Blank Responses Averaged Across All Three Morphs, for the 20 Experimental Morph Sets

\begin{tabular}{|c|c|c|c|c|c|c|c|c|c|c|c|c|c|}
\hline \multirow[b]{2}{*}{$\begin{array}{l}\text { Morph } \\
\text { Group }\end{array}$} & \multirow[b]{2}{*}{$\begin{array}{l}\text { Start- } \\
\text { Point } \\
\text { Name }\end{array}$} & \multicolumn{2}{|c|}{$\begin{array}{c}\text { Start-Point } \\
\text { Morph } \\
\text { (Both Groups) } \\
\end{array}$} & \multicolumn{2}{|c|}{$\begin{array}{c}\text { Midpoint } \\
\text { Morph, When } \\
\text { Start Point } \\
\text { Named First } \\
\end{array}$} & \multicolumn{2}{|c|}{$\begin{array}{c}\text { Midpoint } \\
\text { Morph, When } \\
\text { Named First } \\
\end{array}$} & \multicolumn{2}{|c|}{$\begin{array}{c}\text { Midpoint } \\
\text { Morph, When } \\
\text { End Point } \\
\text { Named First } \\
\end{array}$} & \multicolumn{2}{|c|}{$\begin{array}{c}\text { End-Point } \\
\text { Morph } \\
\text { (Both Groups) } \\
\end{array}$} & \multirow[b]{2}{*}{$\begin{array}{l}\text { End-Point } \\
\text { Name }\end{array}$} & \multirow[b]{2}{*}{$\begin{array}{c}\text { Blank } \\
\text { Responses }\end{array}$} \\
\hline & & $\begin{array}{l}\text { Start- } \\
\text { Point } \\
\text { Name }\end{array}$ & $\begin{array}{l}\text { End- } \\
\text { Point } \\
\text { Name }\end{array}$ & $\begin{array}{l}\text { Start- } \\
\text { Point } \\
\text { Name }\end{array}$ & $\begin{array}{l}\text { End- } \\
\text { Point } \\
\text { Name }\end{array}$ & $\begin{array}{l}\text { Start- } \\
\text { Point } \\
\text { Name }\end{array}$ & $\begin{array}{l}\text { End- } \\
\text { Point } \\
\text { Name }\end{array}$ & $\begin{array}{l}\text { Start- } \\
\text { Point } \\
\text { Name }\end{array}$ & $\begin{array}{l}\text { End- } \\
\text { Point } \\
\text { Name }\end{array}$ & $\begin{array}{l}\text { Start- } \\
\text { Point } \\
\text { Name }\end{array}$ & $\begin{array}{c}\text { End- } \\
\text { Point } \\
\text { Name }\end{array}$ & & \\
\hline 1 & GIRAFFE & 83 & 1 & 47 & 14 & 41 & 24 & 33 & 20 & 1 & 62 & HORSE & 3 \\
\hline 1 & SHARK & 88 & 10 & 71 & 26 & 51 & 48 & 15 & 79 & 6 & 89 & FISH & 0 \\
\hline 1 & BATH & 100 & 0 & 84 & 16 & 70 & 27 & 44 & 54 & 15 & 84 & SINK & 0 \\
\hline 1 & LIZARD & 50 & 44 & 49 & 47 & 9 & 90 & 14 & 82 & 11 & 86 & FROG & 0 \\
\hline 1 & CUP & 98 & 2 & 49 & 48 & 25 & 73 & 6 & 89 & 1 & 97 & JUG & 1 \\
\hline 1 & PIG & 99 & 0 & 95 & 3 & 97 & 2 & 95 & 4 & 11 & 74 & DOG & 0 \\
\hline 1 & CHAIR & 97 & 0 & 74 & 5 & 46 & 18 & 22 & 68 & 1 & 94 & BED & 1 \\
\hline 1 & BENCH & 98 & 0 & 55 & 33 & 50 & 48 & 36 & 57 & 0 & 98 & CHAIR & 0 \\
\hline 1 & STAPLER & 92 & 4 & 71 & 23 & 14 & 62 & 7 & 63 & 2 & 63 & HOLEPUNCH & 6 \\
\hline 1 & LION & 45 & 24 & 35 & 39 & 10 & 65 & 18 & 59 & 10 & 66 & DOG & 2 \\
\hline 2 & CHAIR & 89 & 11 & 77 & 23 & 74 & 25 & 25 & 70 & 8 & 75 & STOOL & 0 \\
\hline 2 & CAMEL & 96 & 0 & 84 & 7 & 54 & 31 & 61 & 23 & 7 & 19 & LLAMA & 1 \\
\hline 2 & SPOON & 87 & 0 & 68 & 1 & 18 & 1 & 14 & 35 & 0 & 85 & KNIFE & 0 \\
\hline 2 & CANOE & 76 & 3 & 44 & 9 & 22 & 16 & 17 & 13 & 2 & 17 & ROWING BOAT & 0 \\
\hline 2 & DOG & 96 & 0 & 68 & 15 & 66 & 11 & 45 & 21 & 2 & 93 & GIRAFFE & 4 \\
\hline 2 & CAR & 100 & 0 & 33 & 51 & 46 & 44 & 18 & 77 & 17 & 77 & VAN & 0 \\
\hline 2 & KEY & 95 & 1 & 70 & 16 & 50 & 24 & 44 & 32 & 0 & 86 & SWORD & 1 \\
\hline 2 & SUBMARINE & 97 & 0 & 83 & 16 & 61 & 39 & 53 & 47 & 16 & 82 & BOAT & 0 \\
\hline 2 & DUCK & 99 & 0 & 87 & 8 & 80 & 8 & 50 & 30 & 20 & 53 & CHICKEN & 0 \\
\hline \multirow[t]{2}{*}{2} & BOTTLE & 70 & 0 & 46 & 4 & 3 & 14 & 1 & 54 & 0 & 91 & WATERINGCAN & 4 \\
\hline & Mean & 88 & 5 & 65 & 20 & 44 & 33 & 31 & 49 & 7 & 75 & & 1 \\
\hline
\end{tabular}

Table 4

Alternative Names Provided by at Least $5 \%$ of Participants for the Start-Point, Midpoint, and End-Point Morphs for the 20 Experimental Morph Sets in Experiment 4

\begin{tabular}{|c|c|c|c|c|c|c|}
\hline $\begin{array}{l}\text { Start- } \\
\text { Point } \\
\text { Name }\end{array}$ & $\begin{array}{l}\text { Start-Point } \\
\text { Morph }\end{array}$ & $\begin{array}{l}\text { Midpoint Morph, } \\
\text { When Start Point } \\
\text { Named First } \\
\end{array}$ & $\begin{array}{l}\text { Midpoint Morph, } \\
\text { When Named First }\end{array}$ & $\begin{array}{l}\text { Midpoint Morph, } \\
\text { When End Point } \\
\text { Named First }\end{array}$ & $\begin{array}{l}\text { End-Point } \\
\text { Morph }\end{array}$ & $\begin{array}{l}\text { End-Point } \\
\text { Name }\end{array}$ \\
\hline GIRAFFE & deer $10 \%$ & deer $24 \%$ & deer $33 \%$ & deer $27 \%$, llama $6 \%$ & deer $14 \%$, moose $6 \%$ & HORSE \\
\hline PIG & & & & & fox $9 \%$ & DOG \\
\hline CHAIR & & seat $7 \%$, couch $5 \%$ & $\begin{array}{l}\text { couch } 13 \% \text {, seat } 7 \% \text {, } \\
\text { sofa } 7 \%\end{array}$ & & & BED \\
\hline BENCH & & seat $10 \%$ & & seat $6 \%$ & & CHAIR \\
\hline STAPLER & & & photocopier 5\% & photocopier $6 \%$ & & HOLEPUNCH \\
\hline LION & $\begin{array}{r}\text { leopard } 7 \% \text {, } \\
\text { puma } 5 \%\end{array}$ & cat $8 \%$ & & animal 6\% & & DOG \\
\hline CHAIR & & & & & $\operatorname{sink} 7 \%$ & STOOL \\
\hline CAMEL & & & & & $\begin{array}{l}\text { horse } 46 \% \text {, deer } 8 \% \text {, } \\
\quad \operatorname{dog} 8 \%\end{array}$ & LLAMA \\
\hline SPOON & trowel $6 \%$ & $\begin{array}{r}\text { trowel } 18 \%, \\
\text { spade } 8 \%\end{array}$ & trowel $39 \%$, spade $36 \%$ & trowel $26 \%$, spade $17 \%$ & spade $5 \%$ & KNIFE \\
\hline CANOE & boat $21 \%$ & boat $46 \%$ & boat $62 \%$ & boat $68 \%$ & boat $80 \%$ & ROWING BOAT \\
\hline DOG & & & & deer $6 \%$, llama $5 \%$ & & GIRAFFE \\
\hline KEY & & saw $8 \%$ & saw $10 \%$ & saw $8 \%$, knife $5 \%$ & & SWORD \\
\hline DUCK & & & bird $6 \%$ & bird $11 \%$, pigeon $7 \%$ & bird $15 \%$, pigeon $9 \%$ & CHICKEN \\
\hline BOTTLE & & $\begin{array}{c}\text { container } 7 \%, \\
\text { oilcan } 6 \%\end{array}$ & $\begin{array}{c}\text { kettle } 11 \% \text {, oilcan } 11 \% \text {, } \\
\text { jug } 9 \% \text {, can } 7 \% \text {, petrol } \\
\text { can } 6 \% \text {, container } 5 \%\end{array}$ & $\begin{array}{l}\text { oilcan } 18 \% \text {, petrol } \\
\text { can } 8 \%\end{array}$ & & WATERINGCAN \\
\hline
\end{tabular}

Note-In Experiment 4, alternative names accepted as equivalent to the start-point and end-point names were: for boat, ship or trawler; camel, dromedary; canoe, kayak; chicken, hen; cup, mug; dog, wolf; fish, piranha; frog, toad; horse, ass, donkey, mule, or pony; lion, lioness or tiger; pig, boar; sink, basin; sword, dagger; submarine, U-boat. In Experiment 4, alternative names accepted for other labels were: for animal, mammal; banjo, mandolin; deer, stag or antelope; moose, elk; spade, shovel; couch, sofa. 
gave another name). The low accuracy for a few objects appeared to be due to item-specific effects. For example, only $19 \%$ correctly named the llama, with $46 \%$ preferring to label it as a horse. This was probably because "llama" is a low-frequency word that is hard to recall. Supporting this suggestion, "llama" (92\%) was clearly preferred over "horse" (3\%) as a label when both were provided as options in the word-picture verification task tested in Experiment 2 (see Table 1). In contrast, the low accuracy for naming the rowing boat $(17 \%)$ was due to people preferring to use a more general label for this stimulus ("boat," at 80\%; see also Experiment 3) rather than because it could not be distinguished from other boats at the subordinate level (accuracy for the rowing boat was $84 \%$ in Experiment 2; see Table 1). Items such as the 1lama and the rowing boat had a disproportionate effect on mean naming accuracy in Experiment 4: The median accuracy was $96 \%$ for naming the start-point morph, and $83 \%$ for the end-point morph.

For the midpoint morph, averaging over the three groups, $47 \%$ chose the start-point name and $34 \%$ chose the end-point name, with only $18 \%$ choosing another name. The midpoint morph was more frequently labeled with the start-point name if participants had previously seen the start-point morph $(65 \%$, vs. $44 \%$ if the midpoint morph was the first one seen and $31 \%$ if the end-point morph was initially seen). Likewise, the midpoint morph was more likely to be labeled with the end-point name if participants had previously seen the end-point morph (49\%, vs. $33 \%$ if the midpoint morph was the first one seen and $20 \%$ if the start-point morph was initially seen). These results are not consistent with the similarity-based contrast effect (Hampton, Estes, \& Simmons, 2005), which would predict the opposite pattern - namely, that the borderline, midpoint morph would more likely be given the end-point name if the start-point object had previously been labeled, and vice versa. An alternative reason for the priming effect that we observed is that the recent production of the name given to the start-point or end-point morph could have increased its availability as a response to the midpoint morph.

These results demonstrate that most of the start-point morphs were named consistently with the start-point name, most of the end-point morphs were named consistently with the end-point name, and the midpoint morphs were named with a roughly equal mixture of the start- and end-point names. These data therefore confirmed that the stimuli selected for the subsequent three experiments were appropriate.

\section{EXPERIMENT 5}

The 20 experimental sets of morphs of familiar objects selected from the first four experiments were now used in three sequential picture-picture matching experiments. These replicated the design of earlier experiments that we had conducted with novel unfamiliar objects (Lawson, 2004b; Lawson \& Bülthoff, 2006). The experiments explored, in two ways, how view sensitivity changed as the difficulty of shape discrimination altered. First, shape discriminability was manipulated within each experiment by varying the size of the shape change that occurred on mismatch trials (Experiments 5 and 6) and on match trials (Experiment 7). Second, shape discriminability was varied across the experiments by varying the task from identification (Experiments 5 and 6) to categorization (Experiment 7).

As discussed in the introduction, there is a consensus among researchers that view sensitivity occurs when shape discrimination is difficult - for example, for subordinatelevel recognition (Biederman \& Gerhardstein, 1995; Hamm \& McMullen, 1998; Lawson \& Bülthoff, 2006; Lawson \& Humphreys, 1996, 1998; Lawson \& Jolicœur, 2003; Tarr \& Cheng, 2003). However, there is considerable disagreement about the point at which this view sensitivity dissipates as shape discrimination becomes easier. Many of the studies investigating the view sensitivity of recognition across rotations in depth have tested people's ability to identify novel objects (e.g., Bülthoff \& Edelman, 1992; Lawson, 2004b; Lawson \& Bülthoff, 2006; Rock \& DiVita, 1987; Tarr, 1995; Vanrie, Béatse, Wagemans, Sunaert, \& Van Hecke, 2002; Vanrie et al., 2001). However, as compared with stimuli from categories that people distinguish between in everyday, basic-level object recognition, these novel stimuli probably differed in terms of their shape discriminability, level of recognition, and shape properties such as complexity and symmetry, as well as in their reduced familiarity, semantic associations, and nameability. Any such differences between novel and familiar objects could have independently influenced the view sensitivity of the novel stimuli, leading to difficulties in drawing conclusions about the view sensitivity of everyday object recognition.

In Experiment 5, we used a sequential picture-picture matching task to test people's ability to ignore view changes while detecting shape changes. Four shapechange and three view-change conditions were tested, which were equivalent to the conditions tested in Experiment 1 of Lawson and Bülthoff (2006). This similarity in experimental designs allowed us to compare performance across familiar and unfamiliar objects. From the results of our previous studies with novel objects (Lawson, 2004b; Lawson \& Bülthoff, 2006; Lawson et al., 2003), in Experiment 5 we expected that view changes would influence people's ability to detect shape changes, even though variation of the views in depth was irrelevant to the task (for contrasting predictions, see Stankiewicz, 2002). We further predicted that greater view sensitivity would be found on same-shape matches than on shape-change mismatches, supporting our hypothesis that view sensitivity increases as the difficulty of shape discrimination increases.

We made one methodological improvement in Experiment 5 relative to Experiment 1 of Lawson and Bülthoff (2006). In Experiment 5, the first picture presented on each trial was the start-point morph for half of the trials and the end-point morph on the remaining trials. In our previous study, the first picture was always the startpoint morph. As a result, it was possible, though unlikely, that in the previous experiment view sensitivity for the 
start-point morph was much greater than for the end-point morph. If so, the pattern of view sensitivity reported there could have been due to systematic variation in the view sensitivity of the different morphs per se, rather than to the difficulty of shape discrimination within the task. This alternative account could not explain any variation in view sensitivity found in Experiment 5.

\section{Method}

Participants. Thirty undergraduate students from the University of Liverpool took part in exchange for course credit.

Materials. Five morphs were presented from each of the 20 experimental morph sets. These were the start-point, midpoint, and end-point morphs, the near-start morph (the one midway between the start- and midpoint morphs; this was S3, S4, or S7, depending on the morphs used as the start and end points for a given morph setsee Experiment 2) and the near-end morph (the one midway between the mid- and end-point morphs; this was S7, S10, or S11). Each morph was, in turn, depicted from three views in depth, at $30^{\circ}, 60^{\circ}$, and $240^{\circ}$. Thus, a total of 15 stimuli were presented for each morph set (five morphs in three views; see Figures 1 and 2 and Table 1). A subset of these pictures (which excluded the near-start and near-end morphs) were shown in Experiments 6 and 7.

Design and Procedure. All participants completed one block of 360 experimental trials. In this block, each of the 20 morph sets was presented on nine matches and nine mismatches. On all trials, both pictures showed morphs from the same morph set. One group of 10 morph sets was presented on half of the trials. Here, the first picture presented was always the $30^{\circ}$ view of the start-point morph. The second picture, on matches, was the same morph, or otherwise was the near-start, midpoint, or end-point morph on hard, medium, and easy shape-change mismatches, respectively. The second group of 10 morph sets was presented on the other half of the trials. Here, the first picture presented was always the $30^{\circ}$ view of the end-point morph. The second picture, on matches, was the same morph, or otherwise was the near-end, midpoint, or start-point morph on hard, medium, and easy shape-change mismatches, respectively. The assignment of morph sets to the first-picture morph (whether startpoint or end-point) was counterbalanced across two subgroups of 15 participants.

Relative to the first picture, the second picture could show a given morph from the same, $30^{\circ}$, view, or from a $60^{\circ}$ or $240^{\circ}$ view. For each of the 20 morph sets, the view-change conditions (i.e., a $0^{\circ}, 30^{\circ}$, or $150^{\circ}$ view change) each included three matches (in which no shape change occurred, since the first and second pictures depicted either the start-point or the end-point morph) and one apiece of the three mismatches - easy, medium, and hard. For generality, we tested two different $\left(30^{\circ}\right.$ and $\left.150^{\circ}\right)$ view changes. Note, though, that there is no straightforward relation between the size of a view change and its effect on the perception of a given stimulus. Some view changes, such as those resulting in foreshortening, make identification much harder. Often, though, there is little effect of increasing the size of a view change above $30^{\circ}-45^{\circ}$ (Foster \& Gilson, 2002; Lawson \& Humphreys, 1996). We therefore did not necessarily expect any difference between the two view-change conditions tested here.

The experiment was run on a Macintosh PowerPC G4 computer using PsyScope (version 1.2.5) experimental presentation software. On each trial, a written cue saying "Get ready for the next trial" appeared for $750 \mathrm{msec}$, and after $500 \mathrm{msec}$, the first picture was presented 50 pixels above and 50 pixels to the right of fixation for $500 \mathrm{msec}$. After a blank interstimulus interval of $400 \mathrm{msec}$, the second picture was presented at fixation until the participant responded. On all trials, the stimulus was thus moved between the first and the second pictures, so performance could not be based on detecting low-level visual changes.

Participants decided whether the two successive pictures showed same- or different-shaped objects and responded, respectively, with a speeded " $\mathrm{m}$ " or " $\mathrm{z}$ " keypress. They were told to ignore any differences in view between the first and second pictures, and they were warned that on mismatches the pictures might show two objects with very similar shapes. After the response, the correct response was given as feedback for $500 \mathrm{msec}$ - that is, the letter " $\mathrm{m}$ " or " $\mathrm{z}$ " was presented at fixation - followed by a 750-msec intertrial interval. The participants took a self-timed break after every 120 trials. Prior to starting the experimental block, they completed a block of 20 practice trials that were selected at random from the experimental trials. The trials were presented in a different random order for each participant.

\section{Results}

ANOVAs were conducted on the mean correct reaction times (RTs) and the percentages of errors for matches and mismatches separately (see Figure 3). Here and in Experiments 6 and 7, the $F$ values in the by-participants and byitems analyses are reported as $F_{\mathrm{p}}$ and $F_{\mathrm{i}}$, respectively. On matches, same-shape " $m$ " responses were correct, whereas on mismatches, shape-change " $z$ " responses were correct. Response latencies less than $300 \mathrm{msec}$ or exceeding $2,300 \mathrm{msec}$ were discarded as errors (less than $2 \%$ of trials). No participants were replaced. There were three empty cells in both the by-participants and by-items ANOVAs. These were filled with the mean for that condition.

All ANOVAs included the within-participants factor of view change $\left(0^{\circ}, 30^{\circ}\right.$, or $\left.150^{\circ}\right)$ and the counterbalancing factor of first-picture morph (start-point or end-point). Here and in Experiments 6 and 7, view sensitivity was similar irrespective of whether the first picture showed a start-point or an end-point morph. The effects of view change that we report were therefore not merely due to greater view sensitivity to certain morphs. Results involving the counterbalancing factor of first-picture morph will not be reported further. Mismatch ANOVAs included the further within-participants factor of shape change (easy, medium, or hard to detect). All pairwise differences noted below were significant $(p<.05)$ in both by-participants and by-items post hoc Newman-Keuls analyses, unless otherwise specified.

Same-shape matches. View change was significant for both RTs $\left[F_{\mathrm{p}}(2,58)=124.47, p<.001 ; F_{\mathrm{i}}(2,38)=\right.$ $132.64, p<.001]$ and errors $\left[F_{\mathrm{p}}(2,58)=166.00, p<\right.$ $\left..001 ; F_{\mathrm{i}}(2,38)=34.47, p<.001\right]$. Same-view matches (723 msec, 6\% errors) were much faster and more accurate than $30^{\circ}$ view changes ( $910 \mathrm{msec}, 34 \%$ errors), which in turn were faster, though not more accurate, than $150^{\circ}$ view changes ( $954 \mathrm{msec}, 33 \%$ errors).

Shape-change mismatches. View change was significant for both RTs $\left[F_{\mathrm{p}}(2,58)=16.42, p<.001 ; F_{\mathrm{i}}(2,38)=\right.$ $9.34, p<.001]$ and errors $\left[F_{\mathrm{p}}(2,58)=31.30, p<.001\right.$; $\left.F_{\mathrm{i}}(2,38)=9.54, p<.001\right]$. In contrast to the same-shape matches, $30^{\circ}$ view-change mismatches $(798 \mathrm{msec}, 24 \%$ errors) were actually more accurate, though no faster, than same-view mismatches (807 msec, 31\%), which in turn were both faster and more accurate than $150^{\circ}$ view-change mismatches ( $858 \mathrm{msec}, 34 \%$; not significant by items for errors in Newman-Keuls analyses).

Shape change was significant for both RTs $\left[F_{\mathrm{p}}(2,58)=\right.$ $\left.38.19, p<.001 ; F_{\mathrm{i}}(2,38)=37.60, p<.001\right]$ and errors $\left[F_{\mathrm{p}}(2,58)=862.04, p<.001 ; F_{\mathrm{i}}(2,38)=151.04, p<\right.$ $.001]$. Large, easy-to-detect shape changes $(747 \mathrm{msec}, 5 \%$ errors) were detected both more quickly and accurately 

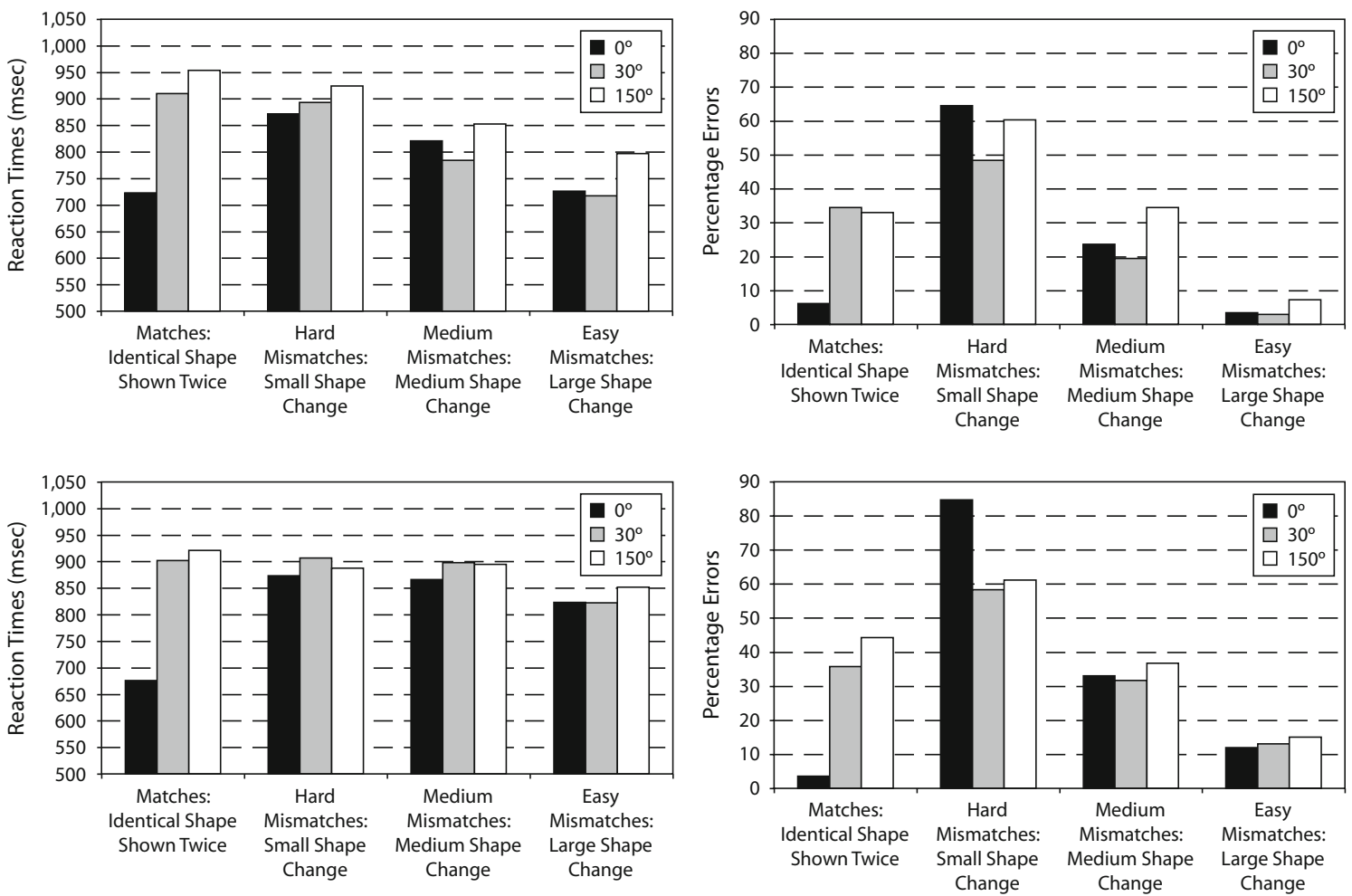

Figure 3. (Top panels) Mean correct reaction times (RTs) and mean percentage errors in Experiment 5 and (bottom panels) mean correct RTs and mean percentage errors in Experiment 1 of Lawson and Bülthoff (2006), for same-view trials $\left(0^{\circ}\right.$ view changes) and view-change trials $\left(30^{\circ}\right.$ or $150^{\circ}$ view changes). On half of the trials in Experiment 5, the first picture was a $30^{\circ}$ view of a start-point morph, and the second picture was either the same start-point morph (on matches, with "different shape" as the wrong response) or the near-start-point, midpoint, or end-point morph from that set (on mismatches, with small, medium, or large shape changes, respectively; here, "different shape" was the correct response). On the other half of the trials, the first picture was a $30^{\circ}$ view of an end-point morph, and the second picture was either the same end-point morph (matches) or the near-end-point, midpoint, or start-point morph from that set (mismatches, with small, medium, or large shape changes, respectively). Lawson and Bülthoff's Experiment 1 used a sequential picture-picture matching task with a design similar to that of Experiment 5.

than medium shape changes ( $819 \mathrm{msec}, 26 \%)$, which in turn were detected both more quickly and accurately than small, hard-to-detect shape changes (897 msec, 58\%).

Finally, the interaction of view change and shape change was not significant for RTs $\left[F_{\mathrm{p}}(4,116)=1.45, p>.2\right.$; $\left.F_{\mathrm{i}}(4,76)=0.38, p>.8\right]$, but it was for errors $\left[F_{\mathrm{p}}(4,116)=\right.$ $\left.10.69, p<.001 ; F_{\mathrm{i}}(4,76)=8.06, p<.001\right]$; see Figure 3 . For errors on hard shape changes, $30^{\circ}$ view changes (48\%) were more accurate than either same-view trials $(65 \%)$ or $150^{\circ}$ view changes $(60 \%)$. For medium shape change errors, both $30^{\circ}$ view changes $(20 \%)$ and same-view trials (24\%) were more accurate than $150^{\circ}$ view changes $(35 \%)$. Finally, view change had no significant effect on errors in detecting easy shape changes, with similarly high accuracies on $30^{\circ}$ view changes (3\%), same-view trials $(4 \%)$, and $150^{\circ}$ view changes $(7 \%)$.

\section{Discussion}

As predicted, there were strong view effects on matches, with same-view trials being much faster and much more accurate than view-change trials. In contrast, view sensitivity was relatively weak on mismatches. This variation in the effects of view change for familiar objects, dependent on the difficulty of a shape discrimination, was similar to that found in Experiment 1 of Lawson and Bülthoff (2006) for novel objects. The pattern of view sensitivity that we previously observed for novel objects thus generalizes to everyday, nameable objects. These results suggest a substantial cost for the achievement of object constancy across depth rotations, even for morphs of familiar objects that must be distinguished in everyday life.

\section{EXPERIMENT 6}

In Experiment 5, all of the participants had to respond to mismatches in a mixture of easy-, medium-, and hardto-detect shape changes, as well as to matches. On every trial, people therefore had to be prepared to detect small, subtle shape changes. The highly view-sensitive performance that we observed on matches in Experiment 5 was probably influenced by this demanding context. When people are recognizing familiar objects, they may typically set more lax criteria for distinguishing between shapes than the criteria that were necessary to perform Ex- 
periment 5 . It is therefore important to determine whether view sensitivity still occurs in a task that does not require difficult shape discrimination, and that may therefore be more similar to everyday object recognition.

To examine this issue, three groups were tested in Experiment 6 . Each group differed only in the difficulty of the shape discrimination required on mismatches, with all groups viewing identical match stimuli. View sensitivity on matches could be compared directly across the groups, since they differed only in the context provided by the mismatches. If performance on matches was viewsensitive, even when shape changes on mismatches were always large and easy to detect, this would suggest that view sensitivity is ubiquitous in everyday object recognition. However, if matches were view-sensitive only when shape discrimination was difficult - that is, for the group who had to detect small shape changes on mismatchesthis would instead indicate that view sensitivity is confined to subordinate-level object recognition.

A second reason for comparing view sensitivity across matches rather than mismatches is that potential problems of interpretation emerge for changes in view sensitivity across mismatches with varying sizes of shape change (as was the case in Experiment 5). In particular, people are less able to detect the occurrence of a view change across pairs of more dissimilar objects (Lawson \& Bülthoff, 2006). For objects with very different shapes (such as an umbrella and an elephant), it becomes almost meaningless to try to specify when views of these two objects are aligned. Note, though, that any effects of this confound would lead to an underestimation of view sensitivity. This issue was avoided in Experiment 6, since our analysis focused on comparing match trials that were identical across the three groups.

A design similar to that of Experiment 6 was used in Lawson (2004b). In this study, morphs of novel objects were presented. The results revealed a strong interaction between view sensitivity on matches and shape discrimination difficulty on mismatches: View sensitivity was much greater when the shape discrimination task was harder. Nevertheless, and importantly, view sensitivity was still found when the shape discrimination context was easythat is, when all mismatches consisted of two completely different shapes. Everyday object recognition is probably much harder than discrimination in this easy context, since most familiar objects have similarly shaped neighbors (e.g., a stool, bench, or table for a chair, or a cat, goat, or sheep for a dog). However, a similar study conducted by Hayward and Williams (2000) produced different results. In their experiment, which also presented novel objects in a sequential picture-picture matching task, they found no change in view sensitivity as the difficulty of shape discrimination increased. Given this discrepancy in the literature, it was important to try to replicate the results of Lawson (2004b) using a different set of stimuli.

\section{Method}

Participants. Thirty-six undergraduate students from the University of Liverpool took part in exchange for course credit.

Design and Procedure. All participants completed one block of 120 trials. Stimuli from each of the 20 morph sets were presented in three matches and three mismatches. For each trial type, we presented the trials in three different view conditions: Relative to the first picture, the second picture was rotated in depth by $0^{\circ}, 30^{\circ}$, or $150^{\circ}$. For the group shown easy-to-detect shape-change mismatches, the stimuli were selected from different morph sets in each of the three view-change conditions.

One group of 10 morph sets was presented on half of the trials. Here, the first picture shown was always the $30^{\circ}$ view of a start-point morph. On matches, the second picture depicted the same start-point morph. On mismatches, the midpoint morph from the same set, the end-point morph from the same set, or the start-point morph from a different set was shown for the hard-, medium-, and easy-to-detect shape-change groups, respectively. The second group of 10 morph sets was presented on the other half of the trials. Here, the first picture shown was always the $30^{\circ}$ view of an end-point morph. On matches, the second picture depicted the same end-point morph. On mismatches, the midpoint morph from the same set, the start-point morph from the same set, or the end-point morph of a different set was shown in the hard-, medium-, and easy-to-detect shape-change groups, respectively.

Twelve participants were assigned to each mismatch shape-change group. Only the mismatches differed across the three groups. The assignment of morph sets to each of the first-picture morphs (whether start-point or end-point) was counterbalanced across two subgroups of 6 participants within each group. The experimental procedure was identical to that of Experiment 5.

\section{Results}

ANOVAs were conducted on the mean correct RTs and on percentages of errors for matches and mismatches separately (see Figure 4). On matches, same-shape "m" responses were correct, whereas on mismatches, shapechange " $z$ " responses were correct. Response latencies less than $275 \mathrm{msec}$ or exceeding 2,300 msec were discarded as errors (less than $1 \%$ of trials). No participants were replaced. There were eight empty cells in the byitems analyses, which were filled by the mean for that condition.

ANOVAs included one within-participants factor, view change $\left(0^{\circ}, 30^{\circ}\right.$, or $\left.150^{\circ}\right)$, and one between-participants factor, mismatch shape change (hard, medium, or easy to detect). There were also two counterbalancing factors, the within-participants factor of first-picture morph (startpoint or end-point) and the between-participants factor of first-picture superset (which group of morph sets provided the start- or end-point morph for the first picture). All pairwise differences noted below were significant $(p<$ .05 ) in by-participants and by-items post hoc NewmanKeuls analyses.

Same-shape matches. View change was significant for both RTs $\left[F_{\mathrm{p}}(2,60)=60.96, p<.001 ; F_{\mathrm{i}}(2,36)=\right.$ $39.76, p<.001]$ and errors $\left[F_{\mathrm{p}}(2,60)=57.85, p<\right.$ $\left..001 ; F_{\mathrm{i}}(2,36)=27.86, p<.001\right]$. Same-view matches (655 msec, $4 \%$ errors) were much faster and more accurate than $30^{\circ}$ view-change matches (744 msec, 19\%), which in turn were faster, but not significantly more accurate, than $150^{\circ}$ view-change matches $(800 \mathrm{msec}, 21 \%)$.

Mismatch shape change was significant for both RTs $\left[F_{\mathrm{p}}(2,30)=23.82, p<.001 ; F_{\mathrm{i}}(2,36)=264.40, p<.001\right]$ and errors $\left[F_{\mathrm{p}}(2,30)=65.69, p<.001 ; F_{\mathrm{i}}(2,36)=84.24\right.$, $p<.001]$. The easy mismatch group (550 msec, $4 \%$ ), who only had to detect large shape changes on mismatches, were much faster and more accurate than the medium mismatch group (731 msec, $10 \%)$, who in turn were much faster and 

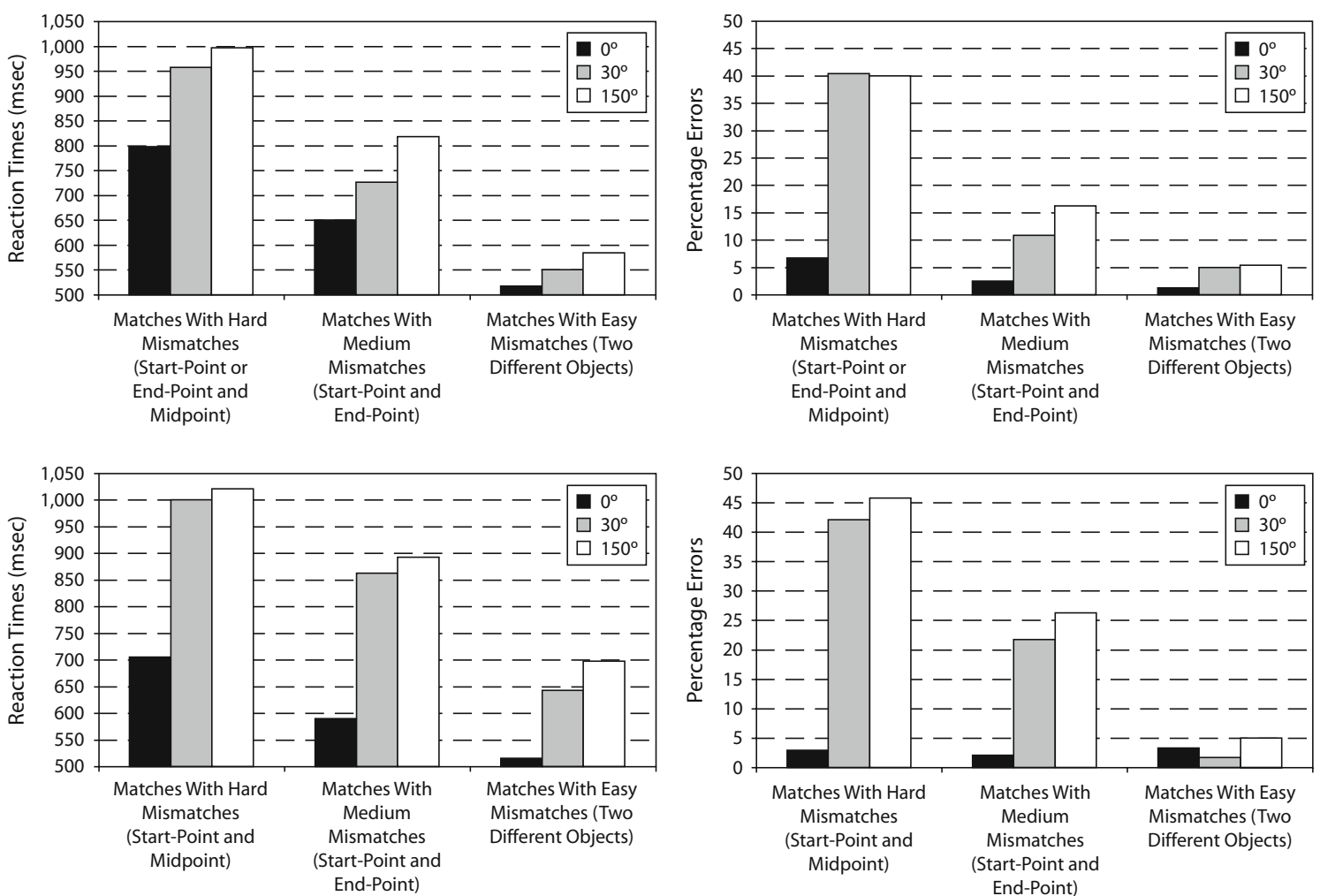

Figure 4. (Top panels) Mean correct reaction times (RTs) and mean percentage errors in Experiment 6 and (bottom panels) mean correct RTs and mean percentage errors in Lawson $(2004 b)$. The results are shown for matches only on same-view trials $\left(0^{\circ}\right.$ view changes) and view-change trials $\left(30^{\circ}\right.$ or $150^{\circ}$ view changes). These trials were identical across the three groups within each experiment, with only the difficulty of mismatches differing across the groups. In Experiment 6 , the first picture was always a $30^{\circ}$ view of a start- or end-point morph. For mismatches in the hard-context group, the second picture showed the midpoint morph from the same morph set. For mismatches in the medium-context group, the second picture showed the end- or start-point morph from the same morph set. For mismatches in the easy-context group, the second picture showed the start- or end-point morph from a different morph set. The study reported by Lawson $(2004 \mathrm{~b})$ used a sequential picture-picture matching task with a design similar to that of Experiment 6.

more accurate than the hard mismatch group $(917 \mathrm{msec}$, $29 \%$ ), who had to detect subtle shape changes on mismatches. Note that these large differences between the three groups are solely due to the mismatch context, since the match trials were identical across all three groups.

Most importantly, the view change $\times$ mismatch shape change interaction was significant for both RTs $\left[F_{\mathrm{p}}(4,60)=\right.$ $\left.6.21, p<.001 ; F_{\mathrm{i}}(4,72)=6.85, p<.001\right]$ and errors $\left[F_{\mathrm{p}}(4,60)=17.96, p<.001 ; F_{\mathrm{i}}(4,72)=16.66, p<.001\right]$; see Figure 4 . For the easy mismatch group, the only significant difference was that same-view matches $(517 \mathrm{msec}$, $1 \%$ errors) were faster than $150^{\circ}$ view-change matches (584 $\mathrm{msec}, 5 \%)$; the speed of responses on $30^{\circ}$ view-change matches fell in between (550 msec, 5\%). View sensitivity was greater for the medium mismatch group, whose sameview matches $(650 \mathrm{msec}, 3 \%)$ were faster than their $30^{\circ}$ view-change matches (726 msec, 11\%), which in turn were faster than the $150^{\circ}$ view-change matches $(818 \mathrm{msec}$, $16 \%$ ). Also, their same-view matches were more accurate than their $150^{\circ}$ view-change matches. View sensitivity was still greater for the hard mismatch group. Here, same-view matches (798 msec, 7\%) were both faster and more accu- rate than matches with either $30^{\circ}(957 \mathrm{msec}, 40 \%)$ or $150^{\circ}$ (997 msec, 40\%) view changes.

Thus, for the easy mismatch group, view-change effects were significant, but only for RTs (not errors), with a difference of just $67 \mathrm{msec}$ (and 4\% errors) between same-view matches and $150^{\circ}$ view-change matches. For the medium mismatch group, view sensitivity was greater between these conditions (168 $\mathrm{msec}, 14 \%$ ) and was significant for both RTs and errors. Finally, view sensitivity increased still further for the hard mismatch group (199 msec, 33\%).

Shape-change mismatches. Mismatches were not the focus of this experiment, since, unlike with the matches, different stimuli were presented to the three groups on these trials. Nevertheless, for completeness, the results are reported here in brief. View change was not significant for RTs $\left[F_{\mathrm{p}}(2,60)=2.27, p>.1 ; F_{\mathrm{i}}(2,36)=1.37, p>.2\right]$, but it was for errors $\left[F_{\mathrm{p}}(2,60)=22.05, p<.001 ; F_{\mathrm{i}}(2,36)=\right.$ $15.80, p<.001]$. Same-view (732 msec, $11 \%$ errors) and $30^{\circ}$ view-change ( $\left.738 \mathrm{msec}, 12 \%\right)$ mismatches were more accurate than $150^{\circ}$ view-change mismatches $(759 \mathrm{msec}$, $22 \%$ ). Mismatch shape change was significant for both 
RTs $\left[F_{\mathrm{p}}(2,30)=16.81, p<.001 ; F_{\mathrm{i}}(2,36)=105.11, p<\right.$ $.001]$ and errors $\left[F_{\mathrm{p}}(2,30)=147.16, p<.001 ; F_{\mathrm{i}}(2,36)=\right.$ $24.11, p<.001]$. The easy mismatch group (570 msec, $4 \%$ ) responded more quickly and accurately than the medium mismatch group ( $762 \mathrm{msec}, 12 \%$ ), who in turn responded more quickly and accurately than the hard mismatch group (896 msec, 30\%). Finally, the view change $X$ mismatch shape change interaction was significant for both RTs $\left[F_{\mathrm{p}}(4,60)=3.82, p<.008 ; F_{\mathrm{i}}(4,72)=2.91\right.$, $p<.03]$ and errors $\left[F_{\mathrm{p}}(4,60)=6.04, p<.001 ; F_{\mathrm{i}}(4,72)=\right.$ $4.89, p<.002]$. This interaction revealed a pattern of view sensitivity similar to, but weaker than, that reported above for matches. There were no view effects for the easy group. For the medium mismatch group, same-view $(8 \%)$ and $30^{\circ}$ view-change $(9 \%)$ mismatches were more accurate than $150^{\circ}$ view-change mismatches $(20 \%)$. For the hard mismatch group, same-view trials (867 $\mathrm{msec}, 23 \%$ ) and $30^{\circ}$ view changes ( $879 \mathrm{msec}, 24 \%)$ were both faster and more accurate than $150^{\circ}$ view changes ( $942 \mathrm{msec}, 43 \%$ ).

Object-specific effects of view changes in Experiments 5 and 6. It is important to establish the generality of the view-sensitive effects reported above across different items. The significant view effects in the by-items analyses of both Experiments 5 and 6 provided evidence that these effects were not just due to performance on a minority of highly view-sensitive objects. This issue was examined in greater detail using data from both experiments. The average cost of compensating for $30^{\circ}$ and $150^{\circ}$ view changes on all matches was calculated for each of the 20 morph sets, individually for the participants in Experiment 5 and for each of the three groups separately in Experiment 6.

There was a striking consistency in the presence of view sensitivity for items across these four groups; see Table 5. In Experiment 5, every one of the 20 morph sets produced better performance on same-view than on view-change trials, for both RTs and errors. For the hard mismatch group in Experiment 6, only one item was an exception to this pattern of view sensitivity, for errors only; for the medium mismatch group, three items were exceptions for RTs only; and for the easy mismatch group, the only exceptions were one item for both RTs and errors, one item for RTs only, and one item for errors only. This level of consistency is particularly impressive given that each of the three groups in Experiment 6 included just 12 participants. Furthermore, of the eight exceptions listed above, four were for the chair-stool morph set, which produced no consistent view-sensitive effects overall, probably in part because the symmetry of the stool meant that its appearance was similar for all views tested. Averaged over size of view change $\left(30^{\circ}\right.$ or $\left.150^{\circ}\right)$, first picture morph (start-point or end-point), and group (Experiment 5 or the hard, medium, or easy mismatch group of Experiment 6), the difference between same-view and view-change trials for the remaining 19 morph sets ranged from 48 to $269 \mathrm{msec}$, for RTs, and $8 \%$ to $42 \%$, for errors. Most importantly, view sensitivity was similar for morph sets belonging to different superordinate categories (such as animals and furniture)

Table 5

Mean Cost of Compensating for View Changes on Match Trials (Averaged Over 30 and $150^{\circ}$ View Changes and Averaged Over First-Picture Morphs) for RTs (Left Five Data Columns) and Errors (Right Five Data Columns) for Each of the 20 Experimental Morph Sets, for All Participants in Experiment 5 (Which Included a Mix of Hard, Medium, and Easy Mismatches), All Three Groups in Experiment 6, and Averaged Over the Four Separate Groups

\begin{tabular}{|c|c|c|c|c|c|c|c|c|c|c|c|}
\hline \multirow{3}{*}{$\begin{array}{c}\text { Morph } \\
\text { Group }+ \\
\text { Level }\end{array}$} & \multirow[b]{3}{*}{$\begin{array}{c}\text { Start- and End- } \\
\text { Point Morph Names }\end{array}$} & \multicolumn{5}{|c|}{ Mean View Effects on RTs (msec) } & \multicolumn{5}{|c|}{ Mean View Effects on Errors (\%) } \\
\hline & & \multicolumn{4}{|c|}{ Mismatch } & \multirow{2}{*}{$\begin{array}{c}\text { Mean } \\
\text { for All } \\
\text { Groups }\end{array}$} & \multicolumn{4}{|c|}{ Mismatch } & \multirow{2}{*}{$\begin{array}{l}\text { Mean } \\
\text { for All } \\
\text { Groups }\end{array}$} \\
\hline & & $\begin{array}{l}\text { Exp 5: } \\
\text { Mixed }\end{array}$ & $\begin{array}{c}\text { Exp 6: } \\
\text { Hard }\end{array}$ & $\begin{array}{l}\text { Exp 6: } \\
\text { Med. }\end{array}$ & $\begin{array}{c}\text { Exp 6: } \\
\text { Easy }\end{array}$ & & $\begin{array}{l}\text { Exp 5: } \\
\text { Mixed }\end{array}$ & $\begin{array}{l}\text { Exp 6: } \\
\text { Hard }\end{array}$ & $\begin{array}{l}\text { Exp 6: } \\
\text { Med. }\end{array}$ & $\begin{array}{c}\text { Exp 6: } \\
\text { Easy }\end{array}$ & \\
\hline 1-Basic & GIRAFFE-HORSE & 295 & 145 & 176 & 60 & 169 & 29 & 54 & 17 & 0 & 25 \\
\hline 1-Sub & SHARK-FISH & 222 & 133 & 149 & 49 & 138 & 23 & 29 & 17 & 8 & 19 \\
\hline 1-Basic & BATH-SINK & 169 & 231 & 48 & 0 & 112 & 24 & 46 & 13 & -8 & 19 \\
\hline 1-Sub & LIZARD-FROG & 338 & 389 & 237 & 112 & 269 & 48 & 58 & 29 & 8 & 36 \\
\hline 1-Basic & CUP-JUG & 198 & 169 & 87 & 130 & 146 & 39 & 50 & 12 & 0 & 25 \\
\hline 1-Basic & PIG-DOG & 267 & 269 & 214 & 48 & 199 & 27 & 17 & 4 & 8 & 14 \\
\hline 1-Basic & CHAIR-BED & 105 & 97 & 160 & 99 & 115 & 3 & 21 & 8 & 8 & 10 \\
\hline 1-Sub & BENCH-CHAIR & 158 & 187 & 189 & 81 & 154 & 15 & 8 & 8 & 0 & 8 \\
\hline 1-Basic & STAPLER-HOLEPUNCH & 256 & 151 & -4 & 15 & 104 & 23 & 42 & 13 & 13 & 23 \\
\hline 1-Basic & LION-DOG & 175 & 235 & 100 & 85 & 149 & 34 & 13 & 21 & 0 & 17 \\
\hline 2-Sub & CHAIR-STOOL & 72 & 44 & -17 & -95 & 1 & 3 & -17 & 4 & -4 & -3 \\
\hline 2-Basic & CAMEL-LLAMA & 235 & 31 & 115 & 22 & 101 & 23 & 46 & 13 & 0 & 20 \\
\hline 2-Basic & SPOON-KNIFE & 188 & 316 & 231 & 119 & 213 & 44 & 58 & 8 & 4 & 29 \\
\hline 2-Sub & CANOE-ROWING BOAT & 160 & 184 & -19 & 81 & 102 & 33 & 38 & 4 & 0 & 19 \\
\hline 2-Basic & DOG-GIRAFFE & 160 & 10 & 18 & 2 & 48 & 7 & 8 & 4 & 13 & 8 \\
\hline 2-Basic & CAR-VAN & 192 & 179 & 108 & 39 & 129 & 22 & 37 & 4 & 4 & 17 \\
\hline 2-Basic & KEY-SWORD & 153 & 85 & 51 & 58 & 87 & 24 & 8 & 0 & 4 & 9 \\
\hline $2-\mathrm{Sub}$ & SUBMARINE-BOAT & 230 & 296 & 238 & 85 & 212 & 63 & 63 & 33 & 4 & 41 \\
\hline 2-Sub & DUCK-CHICKEN & 233 & 125 & 146 & 57 & 140 & 27 & 38 & 4 & 4 & 18 \\
\hline \multirow[t]{2}{*}{ 2-Basic } & BOTTLE-WATERINGCAN & 261 & 118 & 125 & -88 & 104 & 37 & 54 & 4 & 13 & 27 \\
\hline & Mean & 203 & 170 & 118 & 48 & 135 & 28 & 34 & 11 & 4 & 19 \\
\hline
\end{tabular}

Note-Negative values indicate that same-view trials were either slower or less accurate than view-change trials. In the first column the morph group of each morph set is given together with the assumed level (basic or subordinate) of the labels for the start-point and end-point morphs in each morph set (see the typicality rating group in Experiment 3 ). 
and for morph sets with start-point and end-point labels at the basic or the subordinate level (see also Experiment 3). This demonstrates that the strong and consistent view sensitivity observed in our experiments was not confined to a narrow subset of our stimuli.

\section{Discussion}

View sensitivity on matches was found for all three groups tested in Experiment 6. It was relatively weak when shape discrimination was easy, when mismatches always showed large, readily detected shape changes. It was much greater when shape-change detection was more challenging, for the medium, and especially for the hard, mismatch group. This interaction between view sensitivity and the difficulty of shape discrimination replicates the similar finding for novel objects by Lawson (2004b; but see Hayward \& Williams, 2000) and extends it to familiar objects. These results also demonstrate that the pattern of view sensitivity observed in Experiment 5 was not an artifact of either the inclusion of unusually difficult shapechange detection trials within the task or the increased difficulty of determining whether dissimilarly shaped objects were presented from the same view. Instead, view sensitivity was found even for the easy group - for whom shape discrimination was probably much easier than is required in everyday object recognition - and for match trials on which identical shapes were presented, so that view changes would have been readily detected.

\section{EXPERIMENT 7}

In Experiments 5 and 6, shape changes occurred only on mismatches, so people always had to respond "different object" when they detected a shape change. This task is unlike everyday object recognition, in which some shape variation must usually be ignored in order to identify objects as exemplars of a given category. The extent to which exemplars vary in shape within a category differs markedly, but most superordinate-level categories, and even some basic-level categories (e.g., dogs and chairs), include a wide range of shapes. In our final picture-matching experiment, we tested whether performance would still be view-sensitive when people had to do a categorization task in which they had to generalize across shape changes on matches. This task is more like everyday object recognition, in that people had to ignore shape variation on some match trials by responding "same category," even when the two pictures showed objects with different shapes (e.g., the start-point and midpoint morphs from the same set).

We tested the ability of people to achieve view constancy for three levels of difficulty of generalization over shape changes. The task for the first, easy categorization group was most similar to that of everyday, basic-level object recognition. Here, same-category matches were either two identical shapes (the same start-point or end-point morph) or two quite similarly shaped morphs (a start-point and a midpoint morph from the same set or an end-point and a midpoint morph from the same set). The second and third groups were tested on a more challenging task, since some of these same-category matches showed two objects that would usually be given different basic-level names (e.g., a bath and a sink; see Experiment 4 and Table 3). This task required greater shape generalization than does basic-level categorization. In the second, medium categorization group, matches were either two identical shapes or two dissimilar shapes (a start-point and an end-point morph from the same morph set). In the third, hard categorization group, there were no identical shape matches, so people always had to generalize over shape changes on matches-either between a start- or end-point morph and a midpoint morph or between a start-point and an end-point morph. For all three groups, mismatches were always two morphs from different sets, so the context of shape discrimination was easy and was identical to that for the easy group in Experiment 6.

This experiment used the same design as Experiment 2 of Lawson and Bülthoff (2006), which featured pictures of novel objects. In this previous study, view sensitivity was found for all three categorization groups, with better performance on same-view than on view-change matches. However, this view sensitivity was relatively weak, as compared with when the shape discrimination task was harder (e.g., in the study reported in Lawson, 2004b, or in Experiments 5 and 6 here). Given this result, we predicted that performance would be only weakly view-sensitive in Experiment 7.

\section{Method}

Participants. Forty-eight undergraduate students from the University of Liverpool took part in exchange for course credit.

Design and Procedure. The stimuli were identical to those in Experiment 5. All participants completed one block of 240 experimental trials. One group of 10 morph sets was presented on half of the trials. Here, the first picture shown was the $30^{\circ}$ view of a startpoint morph. On matches, the second picture depicted the startpoint, midpoint, or end-point morph from the same set, for zero, moderate, and large shape changes, respectively. On mismatches, the second picture showed the start-point morph from a different morph set. The second group of 10 morph sets was presented on the other half of the trials. Here, the first picture shown was the $30^{\circ}$ view of the end-point morph. On matches, the second picture depicted the end-point, midpoint, or start-point morph from that set, for zero, moderate, and large shape changes, respectively. On mismatches, the second picture showed the end-point morph from a different morph set. The assignment of morph set group to the first-picture morph (whether start-point or end-point) was counterbalanced across two subgroups of 8 participants for each of the three categorization groups.

There were three view conditions: Relative to the first picture, the second picture was depth-rotated by $0^{\circ}, 30^{\circ}$, or $150^{\circ}$. Stimuli from each of the 20 morph sets were shown as the first picture on six matches and six mismatches. Each view condition was presented on two of the six trials of each type; for matches, one of the two trials was assigned to the smaller, and the other to the larger shape-change condition for a given categorization group (see below). Six different morph sets provided the second picture for the six mismatches in which a given morph set provided the first picture.

Sixteen participants were assigned to each of three categorization groups. Only the matches differed across these groups. For the six matches for which a given morph set provided the first picture, the second picture was assigned according to the categorization group: For the easy group, three matches showed the same morph again (so there was zero shape change), and three showed a midpoint morph (producing a moderate shape change). For the medium group, three matches had a zero shape change, and three had 

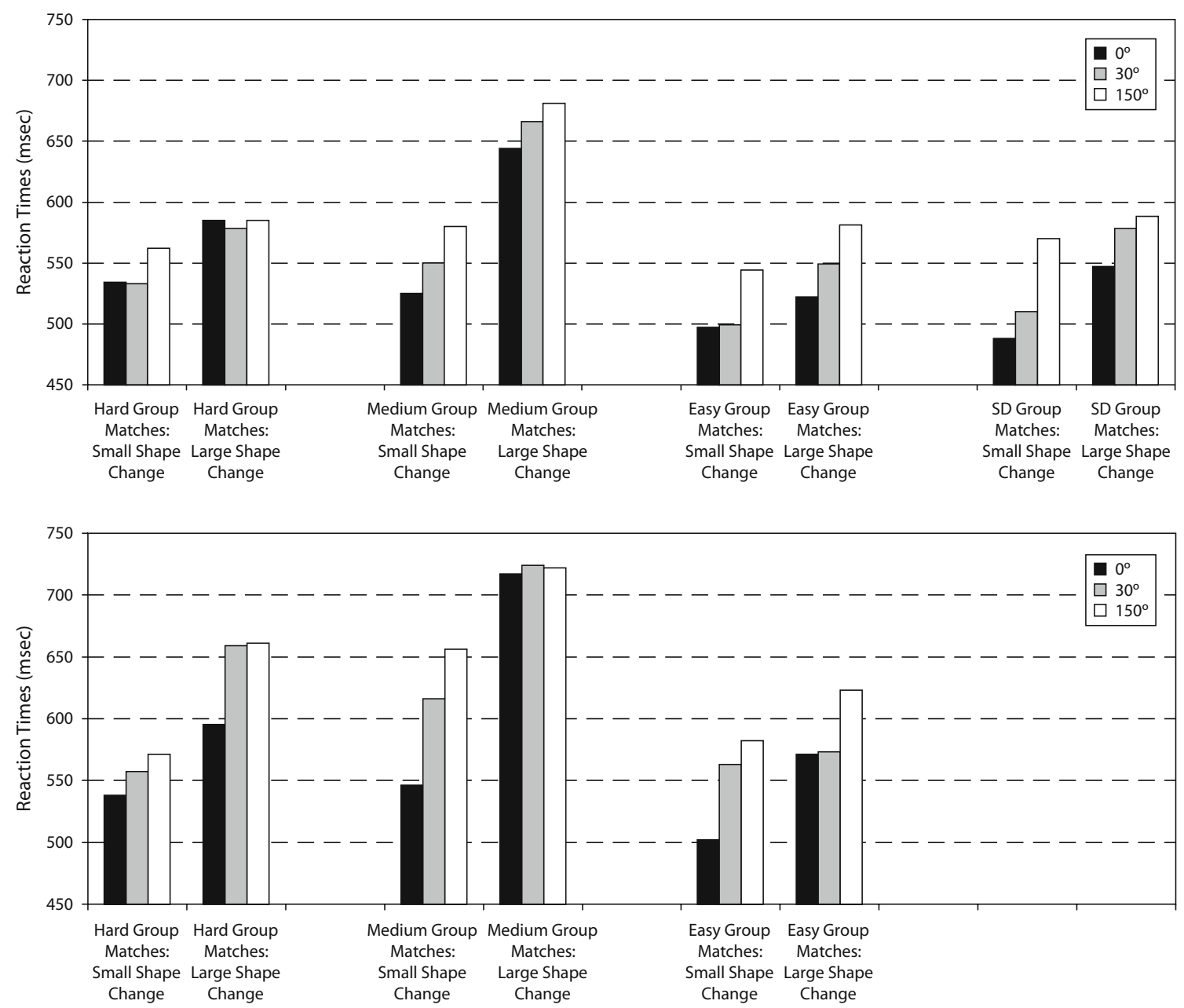

Figure 5A. Mean correct reaction times (RTs) in (top) Experiment 7 and (bottom) Experiment 3 of Lawson and Bülthoff (2006), for the comparable easy, medium, and hard categorization groups in the two studies (not the S0-S3 group of Lawson \& Bülthoff's, 2006, Experiment 3). For Experiment 7 only, the results also include those for a final, similar-distractor (SD) easy categorization group, who saw the same matches as the easy categorization group, but on mismatches were presented with objects shaped similarly to the firstpicture objects. Results are shown for matches only on same-view $\left(0^{\circ}\right.$ view changes) and view-change $\left(30^{\circ}\right.$ or $150^{\circ}$ view changes) trials. In Experiment 7, the first picture was always a $30^{\circ}$ view of a start- or end-point morph. For matches in the easy categorization group, the second picture showed the same start- or end-point morph, for small shape changes, or the midpoint morph from that set, for large shape changes. For matches in the medium categorization group, the second picture showed the same start- or end-point morph, for small shape changes, or the end- or start-point morph from that set, for large shape changes. For matches in the hard categorization group, the second picture showed the midpoint morph from the same set, for small shape changes, or the end- or the start-point morph from the same set, for large shape changes. Lawson and Bülthoff's Experiment 3 used a sequential picture-picture matching task with a design similar to that of Experiment 7.

a large shape change (in which a start-point morph was followed by an end-point morph from the same set, or vice versa). Finally, for the hard group, three matches had a moderate shape change, and three had a large shape change. Note that the moderate and large shape-change matches in Experiment 7 consisted of stimuli identical to, respectively, the medium and large shape-change mismatches in Experiment 5, as well as the small and medium shape-change mismatches in Experiment 6. However, the correct response on these trials had been "different" in the recognition task tested in Experiments 5 and 6, but was now "same" in the categorization task tested in Experiment 7. The procedure was identical to that in Experiments 5 and 6, except that participants were told to decide whether the two successive pictures showed objects from the same or from different categories. The practice trials ensured that participants were aware of the extent of shape variations on matches before they began the experimental trials.

\section{Results}

ANOVAs were conducted on the mean correct RTs and percentages of errors, for matches and mismatches separately; see Figures 5A and 5B. On matches, same-category " $\mathrm{m}$ " responses were correct, whereas on mismatches, category-change " $z$ " responses were correct. Response la- 

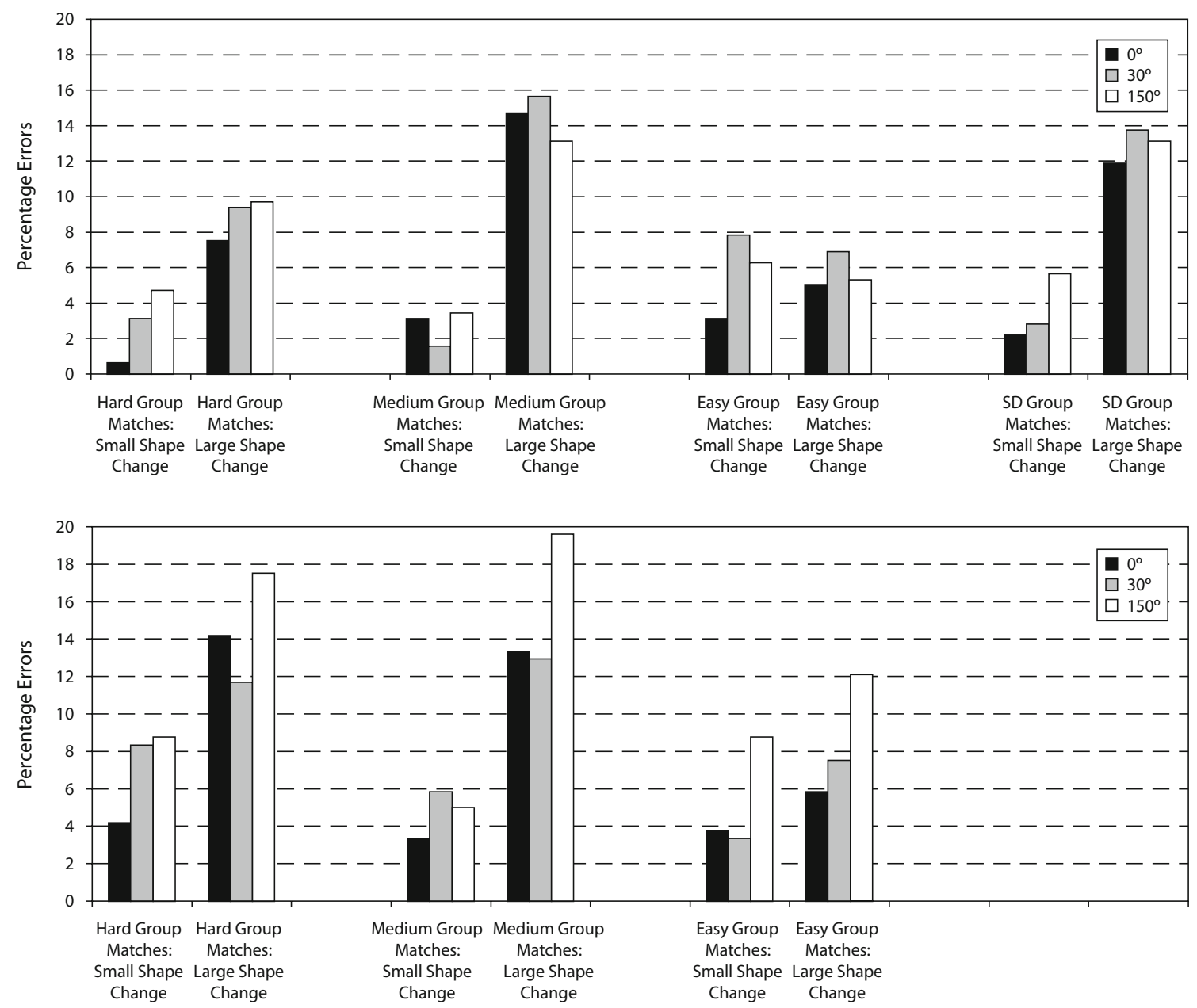

Figure 5B. Mean percentage errors in (top) Experiment 7 and (bottom) Experiment 3 of Lawson and Bülthoff (2006), for the comparable easy, medium, and hard categorization groups in the two studies (not the S0-S3 group of Lawson \& Bülthoff's, 2006, Experiment 3). For Experiment 7 only, the results also include those for a final, similar-distractor (SD) easy categorization group, who saw the same matches as the easy categorization group, but on mismatches were presented with objects shaped similarly to the first-picture objects. Results are shown for matches only on same-view $\left(0^{\circ}\right.$ view changes) and view-change $\left(30^{\circ}\right.$ or $150^{\circ}$ view changes) trials. For more details, see the caption to Figure 5A.

tencies less than $275 \mathrm{msec}$ or exceeding 2,000 msec were discarded as errors (less than 1\% of trials). Two participants were replaced because their overall error rates exceeded $15 \%$. There was one empty cell in the by-items analyses, which was filled with the mean for that condition.

ANOVAs included two within-participants factors, view change $\left(0^{\circ}, 30^{\circ}\right.$, or $\left.150^{\circ}\right)$ and shape change (smaller vs. larger-i.e., zero vs. moderate, zero vs. large, and moderate vs. large shape changes for the easy, medium, and hard categorization groups, respectively). There were also two counterbalancing factors: the within-participants factor of first-picture morph (start-point or end-point) and the between-participants factor of first-picture superset (which group of morph sets provided the start- or end-point morph for the first picture). All pairwise differences noted below were significant $(p<.05)$ in both by-participants and by-items post hoc Newman-Keuls analyses.
Same-category matches. The results here (see Figures $5 \mathrm{~A}$ and $5 \mathrm{~B}$ ) are reported separately for the three categorization groups.

Easy categorization group. View change was significant for both RTs $\left[F_{\mathrm{p}}(2,28)=19.87, p<.001 ; F_{\mathrm{i}}(2,36)=\right.$ $38.48, p<.001]$ and errors, though only marginally for participants $\left[F_{\mathrm{p}}(2,28)=2.51, p<.1 ; F_{\mathrm{i}}(2,36)=4.14\right.$, $p<.03]$. Same-view trials (510 $\mathrm{msec}, 4 \%$ errors $)$ were more accurate than $30^{\circ}$ view changes ( $524 \mathrm{msec}, 7 \%$; not significant for participants) and were faster than $150^{\circ}$ view changes (562 msec, 6\%). Shape change was significant for RTs $\left[F_{\mathrm{p}}(1,14)=10.39, p<.007 ; F_{\mathrm{i}}(1,18)=10.85\right.$, $p<.005]$ but not for errors $\left[F_{\mathrm{p}}(1,14)=0.00, p=1\right.$; $\left.F_{\mathrm{i}}(1,18)=0.00, p=1\right]$. Zero shape changes $(514 \mathrm{msec})$ were categorized faster than moderate shape changes (551 msec). The interaction of view change and shape change was not significant for either RTs $\left[F_{\mathrm{p}}(2,28)=\right.$ 
$\left.1.78, p>.1 ; F_{\mathrm{i}}(2,36)=1.38, p>.2\right]$ or errors $\left[F_{\mathrm{p}}(2,28)=\right.$ $\left.0.52, p>.6 ; F_{\mathrm{i}}(2,36)=1.03, p>.3\right]$.

For the easy categorization group, same-view matches were easier than view-change matches: In particular, they were more accurate than $30^{\circ}$ view changes and faster than $150^{\circ}$ view changes. This view sensitivity replicates the finding of view sensitivity on matches with zero shape change in Experiments 5 and 6. Furthermore, it extends this finding to object categorization, since the same pattern of view sensitivity was found for the moderate shapechange matches. Here, shape generalization was nontrivial, as indicated by the significantly slower responses on moderate than on zero shape-change matches.

Medium categorization group. View change was significant for RTs $\left[F_{\mathrm{p}}(2,28)=9.52, p<.001 ; F_{\mathrm{i}}(2,36)=\right.$ $7.00, p<.003]$ but not for errors $\left[F_{\mathrm{p}}(2,28)=0.10\right.$, $\left.p>.9 ; F_{\mathrm{i}}(2,36)=0.09, p>.9\right]$. Same-view trials (585 msec, $9 \%$ errors) were faster than $30^{\circ}$ view changes (608 msec, 9\%; not significant for items), which in turn were faster than $150^{\circ}$ view changes $(630 \mathrm{msec}, 8 \%)$. Shape change was significant for both RTs $\left[F_{\mathrm{p}}(1,14)=\right.$ $\left.41.81, p<.001 ; F_{\mathrm{i}}(1,18)=54.73, p<.001\right]$ and errors $\left[F_{\mathrm{p}}(1,14)=60.93, p<.001 ; F_{\mathrm{i}}(1,18)=20.22\right.$, $p<.001]$. Zero shape changes (552 msec, 3\% errors) were categorized more quickly and accurately than large shape changes (664 msec, 14\%). The interaction of view change and shape change was not significant for either RTs $\left[F_{\mathrm{p}}(2,28)=0.75, p>.4 ; F_{\mathrm{i}}(2,36)=0.54, p>.5\right]$ or errors $\left[F_{\mathrm{p}}(2,28)=1.11, p>.3 ; F_{\mathrm{i}}(2,36)=1.12\right.$, $p>$.3].

For the medium categorization group, same-view matches were faster than view-change matches. These results replicated the view sensitivity found for zero shapechange trials in the easy categorization group. More importantly, they extended this finding to large shape-change matches. Here, shape generalization was difficult, as indicated by the much slower and less accurate responses, as compared with those to zero shape-change matches.

Hard categorization group. View change was marginally significant for RTs $\left[F_{\mathrm{p}}(2,28)=2.82, p<.08 ; F_{\mathrm{i}}(2,36)=\right.$ $2.51, p<.1]$ and was significant for errors $\left[F_{\mathrm{p}}(2,28)=\right.$ $\left.3.71, p<.04 ; F_{\mathrm{i}}(2,36)=3.44, p<.05\right]$. Same-view trials (559 msec, $4 \%$ errors) were more accurate than $150^{\circ}$ view changes $(573 \mathrm{msec}, 7 \%)$. There were no significant effects involving $30^{\circ}$ view changes $(556 \mathrm{msec}, 6 \%)$. Shape change was significant for both RTs $\left[F_{\mathrm{p}}(1,14)=\right.$ $\left.41.89, p<.001 ; F_{\mathrm{i}}(1,18)=22.51, p<.001\right]$ and errors $\left[F_{\mathrm{p}}(1,14)=35.25, p<.001 ; F_{\mathrm{i}}(1,18)=12.26, p<.003\right]$. Moderate shape changes (543 $\mathrm{msec}, 3 \%$ errors) were categorized more quickly and accurately than large shape changes (583 $\mathrm{msec}, 9 \%)$. The interaction of view change and shape change was significant for RTs, though only marginally so for participants $\left[F_{\mathrm{p}}(2,28)=3.07, p<.07\right.$; $\left.F_{\mathrm{i}}(2,36)=4.16, p<.03\right]$, but it was not significant for errors $\left[F_{\mathrm{p}}(2,28)=0.21, p>.8 ; F_{\mathrm{i}}(2,36)=0.34, p>.7\right]$. For moderate shape changes, same-view trials ( $534 \mathrm{msec}$, $1 \%$ errors) and $30^{\circ}$ view changes (533 $\left.\mathrm{msec}, 3 \%\right)$ were faster than $150^{\circ}$ view changes $(562 \mathrm{msec}, 5 \%)$. In contrast, for large shape changes there were no significant differences between the speeds of same-view trials $(585 \mathrm{msec}$ and $8 \%$ errors), $30^{\circ}$ view changes ( $578 \mathrm{msec}, 9 \%$ ), and $150^{\circ}$ view changes ( $\left.585 \mathrm{msec}, 10 \%\right)$.

For the hard categorization group, same-view matches were more accurate (and, for moderate shape changes, faster) than $150^{\circ}$ view-change matches. This extended the finding of view sensitivity for the easy and medium categorization groups to a case in which shape generalization was difficult and shape changes occurred on every trial (both matches and mismatches).

Category-change mismatches. Mismatch trials were not the focus of theoretical interest here, but they are reported for completeness. There were no significant effects of view change, shape change, or view change $X$ shape change in either the by-participants or by-items ANOVAs for RTs or errors in any of the three groups. Mean RTs (and errors) were $567 \mathrm{msec}(6 \%)$ for the easy categorization group, $635 \mathrm{msec}(6 \%)$ for the medium categorization group, and $588 \mathrm{msec}(5 \%)$ for the hard categorization group.

\section{Discussion}

In Experiment 7, as in Experiments 5 and 6, people performed better on same-view than on view-change trials. This was the case for all three categorization groups. However, view sensitivity was relatively weak. There was a maximum difference of around $50 \mathrm{msec}$ and $5 \%$ errors between same-view and $150^{\circ}$ view-change matches, with similar levels of view sensitivity, across the easy, medium, and hard groups. This may have been because shape discrimination was so easy for all three groups. The two objects shown on mismatches were selected at random and so were usually highly dissimilar in shape to each other. This was like the shape-change discrimination task for the easy context group in Experiment 6 (see Figure 4). In contrast, in everyday object recognition, the visual system must usually distinguish a given object from all possible distractors. The closest of these distractors will often be highly similar in shape to the object. For example, the nearest neighbors in shape space to a goat are animals such as dogs, sheep, horses, and deer. These objects are much more similar in shape to a goat than an object chosen at random would be, so the criteria for detecting shape changes when categorizing the goat may be more stringent.

In order to examine whether the choice of mismatch distractors influenced view sensitivity in Experiment 7 , we tested a final group of 16 participants. This group saw the same matches as the easy categorization group, but the mismatches differed. On mismatches, two similarly shaped objects were presented, with different mismatch distractors being chosen to be paired with each morph set. We tried to choose distractors with shapes similar to the first object presented on mismatch trials. For example, the teapot-wateringcan morphs were used as distractors for the cup-jug morphs. The mismatch distractor manipulation was intended to increase the difficulty of shape discrimination for this group relative to the easy categorization group. We predicted that view sensitivity would be greater for this final, similar-distractor group if increasing the similarity of objects on mismatches made the task harder and more like everyday object recogni- 
tion. This between-group manipulation was similar to that tested across the hard, medium, and easy groups in Experiment 6 . There we found a striking increase in view sensitivity as the difficulty of detecting shape changes in mismatches increased from easy to hard (see Figure 4).

Similar-distractor group tested with the easy categorization task. Sixteen extra participants were tested in the easy categorization condition described above, except that different distractors were presented on mismatches. On mismatches, the second (distractor) object was selected to have a shape similar to the first object shown. These distractors came from the full set of 53 morph sets used in Experiment 1. For example, the pig-dog morph set provided distractors for the giraffe-horse morph set. The data were analyzed in the same way as for the other groups.

View change was significant for RTs $\left[F_{\mathrm{p}}(2,28)=\right.$ $\left.25.86, p<.001 ; F_{\mathrm{i}}(2,36)=32.42, p<.001\right]$ but not for errors $\left[F_{\mathrm{p}}(2,28)=1.33, p>.2 ; F_{\mathrm{i}}(2,36)=1.87\right.$, $p>$.1]. Same-view trials $(518 \mathrm{msec}, 7 \%$ errors $)$ were faster than $30^{\circ}$ view changes (544 msec, $8 \%$ ), which in turn were faster than $150^{\circ}$ view changes $(579 \mathrm{msec}, 9 \%)$. Shape change was significant for both RTs $\left[F_{\mathrm{p}}(1,14)=\right.$ $\left.27.54, p<.001 ; F_{\mathrm{i}}(1,18)=13.30, p<.002\right]$ and errors $\left[F_{\mathrm{p}}(1,14)=18.48, p<.001 ; F_{\mathrm{i}}(1,18)=15.78, p<.001\right]$. Zero shape changes (523 $\mathrm{msec}, 4 \%$ errors) were categorized faster and more accurately than moderate shape changes ( $571 \mathrm{msec}, 13 \%)$. The interaction of view change and shape change was significant for RTs $\left[F_{\mathrm{p}}(2,28)=\right.$ $6.80, p<.004 ; F_{\mathrm{i}}(2,36)=7.43, p<.003$ ] but not for errors $\left[F_{\mathrm{p}}(2,28)=0.64, p>.5 ; F_{\mathrm{i}}(2,36)=1.56, p>.2\right]$ (see Figure 5). With a zero shape change, same-view trials (488 msec, $2 \%$ errors) were faster than $30^{\circ}$ view changes $(510 \mathrm{msec}, 3 \%)$, which in turn were faster than $150^{\circ}$ view changes (570 msec, 6\%). With moderate shape changes, same-view trials ( $547 \mathrm{msec}, 12 \%$ errors) were again faster than $30^{\circ}$ view changes ( $578 \mathrm{msec}, 14 \%$ ), but $30^{\circ}$ view changes were not significantly faster than $150^{\circ}$ view changes ( $588 \mathrm{msec}, 13 \%$ ). For mismatches, the mean RT was $610 \mathrm{msec}$, and there were $8 \%$ errors. Note that mismatches were therefore only $43 \mathrm{msec}$ slower and $2 \%$ less accurate than mismatches for the easy categorization group.

As expected, matches were once again view sensitive for the similar-distractor group. However, their performance only improved modestly on same-view as compared with view-change trials (44 msec faster, $2 \%$ more accurate). In particular, contrary to our predictions, view sensitivity for this group was not significantly greater than that observed for the easy categorization group (33 msec, 3\%). There was no significant group $\times$ view change or group $\times$ view change $X$ shape change interaction when data from both groups were analyzed together.

This lack of a difference in view sensitivity between the two easy categorization groups may have resulted from a failure to substantially increase the difficulty of shape discrimination in the similar-distractor group. Here, the same morph set (e.g., teapot-wateringcan) provided all of the mismatch distractors for a given morph set (e.g., cupjug). These mismatches were therefore less variable than those for the easy categorization group, in which morphs from six different sets appeared in the six mismatches for a given morph set. This increased predictability of mismatches for the similar-distractor group could have made shape discrimination easier than for the easy categorization group, which in turn might have offset any increase in the difficulty of shape discrimination produced by the greater similarity of the two objects shown on mismatches. A second possible reason for the result is that it was not possible to pair all of the experimental morph sets with similarly shaped morph sets, given that only 53 morph sets were available for use as distractors. We have direct evidence that shape discrimination difficulty was not much greater for the similar-distractor group: Their overall performance was both fast and accurate, and it was little worse than that for the easy categorization group, both for matches (15 msec slower, $2 \%$ more errors) and mismatches (40 msec slower, $2 \%$ more errors). In contrast, in Experiment 6 , the shape discrimination manipulation produced a much larger difference between the medium and easy mismatch shape-change groups, both for matches (181 msec, $6 \%$ ) and mismatches (192 msec, 8\%).

The shape discrimination task in Experiment 7 was probably easier than everyday object recognition. For the easy, medium, and hard categorization groups, most mismatches involved a large shape change, whereas for the similar-distractor group the mismatches repeatedly presented the same distractor paired with a given object, and this distractor was often not very similar in shape to that object. In contrast, most familiar objects that we have to recognize in our daily lives must be distinguished from a range of similarly shaped objects (e.g., a chair from a stool, table, or sofa). Nevertheless, despite shape discrimination in this experiment being as easy or easier than that required for everyday recognition, all four groups still produced view-sensitive performance (see Figure 5), which suggests that basic-level categorization of familiar objects is view-sensitive.

\section{GENERAL DISCUSSION}

First, the results of the three sequential matching experiments reported here suggest that the recognition and categorization of familiar objects is normally sensitive to the view from which an object is seen. The achievement of object constancy over depth rotation thus appears to incur a cost under most circumstances, including - most importantly - when familiar objects are identified at the basic level in everyday life. Second, the benefit of seeing an object from the same orientation in depth in which it has previously been identified is modulated by the overall difficulty of shape discrimination; the advantage for same-view over view-change matches is substantial when shape change detection is difficult, but reduced when shape discrimination is easy. This was the case for matches relative to mismatches in Experiment 5, and for matches in the context of small relative to large shapechange mismatches in Experiment 6. Third, the magnitude and pattern of view sensitivity are similar for both the familiar objects tested here and the novel objects tested in 
our earlier studies (see Figures 3, 4, and 5). In particular, we found no evidence that view-sensitive performance is restricted to the recognition and categorization of novel objects. Together, these results suggest that view-specific information is encoded as an integral part of the representations of a wide range of objects. These include familiar objects from a diverse range of superordinate categories (see Table 5), with similar view sensitivity for subordinate and basic-level categories of objects (see Table 2). Our results also demonstrate systematic variation in the level of this view sensitivity that is dependent on both the difficulty of shape discrimination and the task. These findings support the hypothesis that view-sensitive representations are typically used when recognizing familiar objects at the basic level, as well as at the subordinate level (Tarr \& Bülthoff, 1995; Tarr \& Cheng, 2003; but see Biederman \& Gerhardstein, 1993, 1995).

Our results are broadly consistent with recent theoretical accounts proposing that both view-specific and view-invariant representations play major roles in object recognition (Foster \& Gilson, 2002; Hummel, 2001; Tarr $\&$ Bülthoff, 1995, 1998). For example, Hummel and colleagues (Hummel, 2001; Hummel \& Stankiewicz, 1998; Thoma, Davidoff, \& Hummel, 2007; Thoma et al., 2004) have suggested that two types of shape representations can mediate object recognition. These representations work in parallel and comprise a view-specific, shape-specific, holistic representation that is sensitive to depth rotation, and an analytic representation that can generalize over some view and shape changes. The former representation can be activated automatically and may dominate when object recognition is easy, whereas the latter representation requires attention and is slower to be activated, but it is necessary for generalizing over either view or shape changes. The view-specific representation could mediate the same-view, same-shape matches in Experiments 5, 6 , and 7 , whereas only the analytic representation could support generalization across view and shape changes on the remaining trials. This paradigm could account for the generally superior performance on same-view, sameshape matches in our research. However, it is not clear whether the view-specific representations could also mediate same-view, shape-change matches in Experiment 7. If not, this account would fail to explain why view sensitivity was also found on these trials.

A potential critique of the present study is its use of a sequential picture-picture matching task. A particular issue with this task is that identical pictures were presented on same-view, same-shape trials. On such trials, a simple similarity matching strategy might suffice to determine that a picture had been repeated, but this strategy would be useless for everyday object recognition. Three points can be made regarding this concern. First, in Experiments 5, 6 , and 7 , the second picture was always presented at a different position from the first, altering the retinal input and thus preventing the use of very low-level matching strategies. Second, a simple matching strategy would fail for those same-view, same-category trials in Experiment 7 in which the first and second pictures showed different shapes. However, view sensitivity was similar on these moderate and large shape-change matches to that on the zero shape-change matches, in which identical pictures were presented. This suggests that people did not benefit substantially from a simple matching strategy for the identical-repetition trials. Third, in Experiment 6 (see Figure 4) there were striking differences in performance on the view-change matches across the three groups tested. These trials were the same for all three groups, and the differences cannot be due to simple, low-level similarity matching, because there was always a view change (hence, a picture change) on these trials. This result demonstrates that shape discriminability influences view sensitivity independent of the results from same-view (identicalrepetition) matches. Some researchers have argued that tasks such as sequential matching may be contaminated by familiarity effects, and that naming is a better task for examining object recognition (e.g., Biederman \& Gerhardstein, 1993). However, many others disagree (e.g., Tarr \& Bülthoff, 1995). Furthermore, note that naming is a relatively noisy, unreliable task (Lawson, 2004a), making it a poor choice for examining the complex interactions investigated here. A final issue with the sequential matching task is that it does not necessitate the involvement of longterm object representations. This is, in fact, a strength of the task, since for this reason it can be used with novel as well as familiar objects. Furthermore, previous research has demonstrated that sequential matching tasks are sensitive to effects of object familiarity and perceptual training (e.g., X. Jiang et al., 2007; Lawson \& Humphreys, 1996). Notwithstanding these points, it would be useful to extend the research reported here to a wider range of tasks.

The present results demonstrate the importance of shape discriminability and task in modulating view sensitivity, but current accounts of the achievement of object constancy pay little attention to these factors. In particular, we suggest that the difficulty of shape discrimination may be a much more important factor, when determining whether performance is view-sensitive, than the identification level of a category (subordinate or basic; Rosch et al., 1976). However, most researchers have focused on the latter factor. These two effects are often confounded: Subordinate-level distinctions (e.g., between different breeds of dog) usually require finer shape discriminations than basic-level categorization (e.g., between different animals). Nevertheless, it is possible to tease apart these two factors, as was done here.

An important benefit of manipulating shape discriminability directly is that this factor is relatively easy to specify objectively. In contrast, trying to determine whether a given category label is at the subordinate, basic, or superordinate level can be time-consuming, subjective, and contentious. For example, at what level are the following labels, and to which superordinate category do they belong: "telephone," "stapler," "vase," "steering wheel," "angel," "pillow," "tooth," "brick," "umbrella," "leaf," "feather," "mould," "ladder," and "button" (see Lawson \& Jolicœur, 2003)? Furthermore, atypical exemplars of a category are often named not at the basic but at the subordinate level (e.g., "penguin," not "bird"; see Jolicœur et al., 1984; Murphy \& Brownell, 1985; Op de Beeck \& 
Wagemans, 2001). It seems likely that one important reason why penguins are normally identified at the subordinate level is that they are dissimilar in shape from other exemplars of both the category of birds and other categories. Note, though, that this difference in 3-D shape is often confounded with other, semantic factors (e.g., unlike most other birds, penguins swim, do not build nests, and cannot fly) and with people's knowledge and experience of a category (Medin \& Atran, 2004).

In conclusion, the empirical findings presented here map out variation in the difficulty the human visual system has in achieving object constancy across depth rotations over a wide range of conditions. Performance was superior on same-view relative to view-change trials across a diverse range of conditions. The factors manipulated included the superordinate category and geometry of objects, their familiarity and shape discriminability, and the task. These results provide an important set of constraints on theoretical accounts of object recognition. In particular, they point to the crucial and underappreciated role of shape discriminability in modulating view sensitivity in the recognition of both familiar and novel categories of objects.

\section{AUTHOR NOTE}

This research was supported by a fellowship to the first author from the Economic and Social Research Council (RES-000-27-0162). We gratefully acknowledge the support of the Max-Planck-Institut für biologische Kybernetik in Germany. Correspondence relating to this article may be sent to R. Lawson, School of Psychology, University of Liverpool, Eleanor Rathbone Building, Bedford Street South, Liverpool L69 7ZA, England (e-mail: rlawson@liverpool.ac.uk).

\section{REFERENCES}

Andrews, T. J., \& EwBAnK, M. P. (2004). Distinct representations for facial identity and changeable aspects of faces in the human temporal lobe. Neurolmage, 23, 905-913.

Benton, C. P., Jennings, S. J., \& Chatting, D. J. (2006). Viewpoint dependence in adaptation to facial identity. Vision Research, 46, 3313-3325.

BIEDERMAN, I. (1987). Recognition-by-components: A theory of human image understanding. Psychological Review, 94, 115-147.

Biederman, I., \& Gerhardstein, P. C. (1993). Recognizing depthrotated objects: Evidence and conditions for three-dimensional viewpoint invariance. Journal of Experimental Psychology: Human Perception \& Performance, 19, 1162-1182.

Biederman, I., \& Gerhardstein, P. C. (1995). Viewpoint-dependent mechanisms in visual object recognition: Reply to Tarr and Bülthoff (1995). Journal of Experimental Psychology: Human Perception \& Performance, 21, 1506-1514.

Bülthoff, H. H., \& Edelman, S. (1992). Psychophysical support for a two-dimensional view interpolation theory of object recognition. Proceedings of the National Academy of Sciences, 89, 60-64.

Burgund, E. D., \& MarsoleK, C. J. (2000). Viewpoint-invariant and viewpoint-dependent object recognition in dissociable neural subsystems. Psychonomic Bulletin \& Review, 7, 480-489.

Demeyer, M., Zaenen, P., \& Wagemans, J. (2007). Low-level correlations between object properties and viewpoint can cause viewpointdependent object recognition. Spatial Vision, 20, 79-106.

Eger, E., Schweinberger, S. R., Dolan, R. J., \& Henson, R. N. (2005) Familiarity enhances invariance of face representations in human ventral visual cortex: fMRI evidence. NeuroImage, 26, 1128-1139.

FANG, F., \& HE, S. (2005). Viewer-centered object representation in the human visual system revealed by viewpoint aftereffects. Neuron, $\mathbf{4 5}$, 793-800.

Foster, D. H., \& Gilson, S. J. (2002). Recognizing novel three- dimensional objects by summing signals from parts and views. Proceedings of the Royal Society of London B, 269, 1939-1947.

Gelman, S. A. (2003). The essential child: Origins of essentialism in everyday thought. Oxford: Oxford University Press.

Grill-Spector, K., Kushnir, T., Edelman, S., Avidan, G., ItzchaK, Y., \& MALACH, R. (1999). Differential processing of objects under various viewing conditions in the human lateral occipital complex. Neuron, 24, 187-203.

Hamm, J. P., \& McMullen, P. A. (1998). Effects of orientation on the identification of rotated objects depend on the level of identity. Journal of Experimental Psychology: Human Perception \& Performance, 24, 413-426.

Hampton, J. A., Estes, Z., \& Simmons, C. L. (2005). Comparison and contrast in perceptual categorization. Journal of Experimental Psychology: Learning, Memory, \& Cognition, 31, 1459-1476.

HAYWARD, W. G. (1998). Effects of outline shape in object recognition. Journal of Experimental Psychology: Human Perception \& Performance, 24, 427-440.

HAYWARD, W. G. (2003). After the viewpoint debate: Where next in object recognition? Trends in Cognitive Sciences, 7, 425-427.

HAYWARD, W. G., \& TARR, M. J. (1997). Testing conditions for viewpoint invariance in object recognition. Journal of Experimental Psychology: Human Perception \& Performance, 23, 1511-1521.

Hayward, W. G., \& Williams, P. (2000). Viewpoint dependence and object discriminability. Psychological Science, 11, 7-12.

Hummel, J. E. (2001). Complementary solutions to the binding problem in vision: Implications for shape perception and object recognition. Visual Cognition, 8, 489-517.

Hummel, J. E., \& Stankiewicz, B. J. (1998). Two roles for attention in shape perception: A structural description model of visual scrutiny. Visual Cognition, 5, 49-79.

Jiang, F., Blanz, V., \& O'Toole, A. J. (2007). The role of familiarity in three-dimensional view-transferability of face identity adaptation. Vision Research, 47, 525-531.

Jiang, X., Bradley, E., Rini, R. A., Zeffiro, T., VanMeter, J., \& RieSENHUBER, M. (2007). Categorization training results in shape- and category-selective human neural plasticity. Neuron, 53, 891-903.

Joliceeur, P., Gluck, M. A., \& Kosslyn, S. M. (1984). Pictures and names: Making the connection. Cognitive Psychology, 16, 243-275.

KeIL, F. C. (1989). Concepts, kinds, and cognitive development. Cambridge, MA: MIT Press, Bradford Books.

KeIL, F. C. (2003). Categorisation, causation, and the limits of understanding. Language \& Cognitive Processes, 18, 663-692.

LAwson, R. (1999). Achieving visual object constancy across plane rotation and depth rotation. Acta Psychologica, 102, 221-245.

LAWSON, R. (2004a). Depth rotation and mirror-image reflection reduce affective preference as well as recognition memory for pictures of novel objects. Memory \& Cognition, 32, 1170-1181.

LAwsON, R. (2004b). View sensitivity increases for same-shape matches if mismatches show pairs of more similar shapes. Psychonomic Bulletin \& Review, 11, 896-902.

LAWSON, R. (2008). A comparison of the effects of depth rotation on visual and haptic 3-D object recognition. Manuscript submitted for publication.

Lawson, R., \& Bülthoff, H. H. (2006). Comparing view sensitivity in shape discrimination with shape sensitivity in view discrimination. Perception \& Psychophysics, 68, 655-673.

Lawson, R., Bülthoff, H. H., \& Dumbell, S. (2003). Interactions between view changes and shape changes in picture-picture matching. Perception, 32, 1465-1498.

Lawson, R., \& Humphreys, G. W. (1996). View specificity in object processing: Evidence from picture matching. Journal of Experimental Psychology: Human Perception \& Performance, 22, 395-416.

LaWson, R., \& Humphreys, G. W. (1998). View-specific effects of depth rotation and foreshortening on the initial recognition and priming of familiar objects. Perception \& Psychophysics, 60, 1052-1066.

Lawson, R., \& Humphreys, G. W. (1999). The effects of view in depth on the identification of line drawings and silhouettes of familiar objects: Normality and pathology. Visual Cognition, 6, 165-195.

Lawson, R., Humphreys, G. W., \& Watson, D. G. (1994). Object recognition under sequential viewing conditions: Evidence for viewpointspecific recognition procedures. Perception, 23, 595-614. 
LAWSON, R., \& JolicceUR, P. (2003). Recognition thresholds for planerotated pictures of familiar objects. Acta Psychologica, 112, 17-41.

MARr, D. (1982). Vision: A computational investigation into the human representation and processing of visual information. San Francisco: Freeman.

Medin, D. L., \& Atran, S. (2004). The native mind: Biological categorization and reasoning in development and across cultures. Psychological Review, 111, 960-983.

MurPhy, G. L. (2002). The big book of concepts. Cambridge, MA: MIT Press, Bradford Books.

Murphy, G. L., \& Brownell, H. H. (1985). Category differentiation in object recognition: Typicality constraints on the basic category advantage. Journal of Experimental Psychology: Learning, Memory, \& Cognition, 11, 70-84.

Op de BeEcK, H., \& Wagemans, J. (2001). Visual object categorisation at distinct levels of abstraction: A new stimulus set. Perception, 30, 1337-1361.

Pourtois, G., Schwartz, S., Seghier, M. L., Lazeyras, F., \& VuilLEUMIER, P. (2005). View-independent coding of face identity in frontal and temporal cortices is modulated by familiarity: An event-related fMRI study. NeuroImage, 24, 1214-1224.

Rock, I., \& DiViTA, J. (1987). A case of viewer-centered object perception. Cognitive Psychology, 19, 280-293.

Rosch, E., Mervis, C. B., Gray, W. D., Johnson, D. M., \& BoyesBraem, P. (1976). Basic objects in natural categories. Cognitive Psychology, 8, 382-439.

RYU, J.-J., \& ChAUDHURI, A. (2006). Representations of familiar and unfamiliar faces as revealed by viewpoint-aftereffects. Vision Research, 46, 4059-4063.

SRINIVAS, K. (1995). Representation of rotated objects in explicit and implicit memory. Journal of Experimental Psychology: Learning, Memory, \& Cognition, 21, 1019-1036.

STANKIEWICZ, B. J. (2002). Empirical evidence for independent dimensions in the visual representation of three-dimensional shape. Journal of Experimental Psychology: Human Perception \& Performance, 28, 913-932.

TARR, M. J. (1995). Rotating objects to recognize them: A case study on the role of viewpoint dependency in the recognition of threedimensional objects. Psychonomic Bulletin \& Review, 2, 55-82.
TARR, M. J., \& BÜLTHOFF, H. H. (1995). Is human object recognition better described by geon structural descriptions or by multiple views? Comment on Biederman and Gerhardstein (1993). Journal of Experimental Psychology: Human Perception \& Performance, 21, 1494-1505.

TARR, M. J., \& BüLTHOFF, H. H. (1998). Image-based object recognition in man, monkey and machine. Cognition, 67, 1-20.

TARr, M. J., \& Cheng, Y. D. (2003). Learning to see faces and objects. Trends in Cognitive Sciences, 7, 23-30.

TARr, M. J., \& Pinker, S. (1990). When does human object recognition use a viewer-centered reference frame? Psychological Science, 1, 253-256.

Thoma, V., \& Davidoff, J. (2006). Priming of depth-rotated objects depends on attention and part changes. Experimental Psychology, 53, 31-47.

Thoma, V., Davidoff, J., \& Hummel, J. E. (2007). Priming of planerotated objects depends on attention and view familiarity. Visual Cognition, 15, 179-210.

Thoma, V., Hummel, J. E., \& Davidoff, J. (2004). Evidence for holistic representations of ignored images and analytic representations of attended images. Journal of Experimental Psychology: Human Perception \& Performance, 30, 257-267.

TJan, B. S., \& LEGge, G. E. (1998). The viewpoint complexity of an object-recognition task. Vision Research, 38, 2335-2350.

Vanrie, J., Béatse, E., Wagemans, J., Sunaert, S., \& Van Hecke, P. (2002). Mental rotation versus invariant features in object perception from different viewpoints: An fMRI study. Neuropsychologia, 40, 917-930.

Vanrie, J., Willems, B., \& Wagemans, J. (2001). Multiple routes to object matching from different viewpoints: Mental rotation versus invariant features. Perception, 30, 1047-1056.

Vuilleumier, P., Henson, R. N., Driver, J., \& Dolan, R. J. (2002). Multiple levels of visual object constancy revealed by event-related fMRI of repetition priming. Nature Neuroscience, 5, 491-499.

WiLsOn, K. D., \& FARAH, M. J. (2006). Distinct patterns of viewpointdependent BOLD activity during common-object recognition and mental rotation. Perception, 35, 1351-1366.

(Manuscript received June 5, 2007; revision accepted for publication February 3, 2008.) 